MATHEMATICS OF COMPUTATION

Volume 74, Number 252, Pages 1871-1893

S 0025-5718(05)01755-2

Article electronically published on March 3, 2005

\title{
STRONG TRACTABILITY OF INTEGRATION USING SCRAMBLED NIEDERREITER POINTS
}

\author{
RONG-XIAN YUE AND FRED J. HICKERNELL
}

\begin{abstract}
We study the randomized worst-case error and the randomized error of scrambled quasi-Monte Carlo (QMC) quadrature as proposed by Owen. The function spaces considered in this article are the weighted Hilbert spaces generated by Haar-like wavelets and the weighted Sobolev-Hilbert spaces. Conditions are found under which multivariate integration is strongly tractable in the randomized worst-case setting and the randomized setting, respectively. The $\varepsilon$-exponents of strong tractability are found for the scrambled Niederreiter nets and sequences. The sufficient conditions for strong tractability for Sobolev spaces are more lenient for scrambled QMC quadratures than those for deterministic QMC net quadratures.
\end{abstract}

\section{INTRODUCTION}

Base $b$ scrambling quadrature proposed by Owen 13 is a hybrid method of Monte Carlo (MC) and quasi-Monte Carlo (QMC) methods of integration based on random permutations of the digits of the points in a net or a sequence. Two important properties of this randomization are (i) a scrambled version of a point in the unit cube has the uniform distribution on the unit cube and (ii) a scrambled $(t, m, d)$-net or $(t, d)$-sequence in base $b$ is still a $(t, m, d)$-net or a $(t, d)$-sequence with probability one. Therefore, the scrambled QMC rule

$$
Q_{n, d}\left(f,\left\{\mathbf{x}_{i}\right\}\right)=\frac{1}{n} \sum_{i=1}^{n} f\left(\mathbf{x}_{i}\right)
$$

provides an unbiased estimate of the integral over the unit cube $[0,1)^{d}$,

$$
I_{d}(f)=\int_{[0,1)^{d}} f(\mathbf{x}) d \mathbf{x} .
$$

By using low discrepancy points, this method can achieve superior accuracy to simple MC methods based on independent random points. On the other hand, the random scrambling allows error estimation by replication as in other MC methods. There have been investigations of the variance of $Q_{n, d}$ (by Owen [14, 15], [16], Yue

Received by the editor November 24, 2003 and, in revised form, July 6, 2004.

2000 Mathematics Subject Classification. Primary 65C05, 65D30.

Key words and phrases. Multivariate integration, quasi-Monte Carlo methods, nets and sequences, scrambling.

This work was partially supported by Hong Kong Research Grants Council grant HKBU/2020/02P, National Science Foundation of China grant 10271078, E-Institute of Shanghai Municipal Education Commission (E03004), and the Special Funds for Major Specialties of the Shanghai Education Committee. 
[26, and Yue and Mao [27]), the root mean square discrepancies of the scrambled nets and sequences (by Hickernell [2, Hickernell and Hong [3], and Hickernell and Yue [8]), and error analysis of scrambled QMC quadrature rules in the worst-case, random-case, and average-case settings (by Heinrich, Hickernell and Yue [1]).

Recently, the tractability of integration and approximation based on scrambled QMC algorithms, i.e., QMC tractability, was considered by Yue and Hickernell [25] for the Hilbert spaces spanned by Haar wavelets in base 2. Conditions were found under which integration and approximation are tractable. However, the approach used there is nonconstructive.

The main purpose of this article is to find sufficient conditions for QMC strong tractability for multivariate integration using scrambled Niederreiter points (10, [11) in the randomized worst-case setting and the randomized setting, respectively. We consider two linear spaces of functions. One is the weighted Hilbert space spanned by multidimensional Haar-like wavelets, $\mathcal{H}_{d, \eta, \boldsymbol{\beta}}^{\text {wav }}$. This space is defined in terms of weights $\boldsymbol{\beta}=\left(\beta_{1}, \ldots, \beta_{d}\right)$ which moderate the behavior of functions with respect to successive coordinates. The positive parameter, $\eta$, controls the digital smoothness of functions. The other space is the weighted SobolevHilbert space, $\mathcal{H}_{d, \gamma}^{\mathrm{SH}}$, which is defined in terms of weights $\gamma=\left(\gamma_{1}, \ldots, \gamma_{d}\right)$. These spaces are described in the next section. The tractability problem for deterministic QMC quadrature in weighted Sobolev spaces and Korobov spaces has been studied in the worst-case settings by Hickernell and Woźniakowski [6, 7, Sloan and Woźniakowski [17, 18, Hickernell and Wang [4, and Wang [22, 23. In Sloan and Woźniakowski [18, 19] the tractability problem of classical MC quadrature is also considered for weighted Korobov spaces in randomized settings.

There are several reasons for the choice of quadrature rule, spaces, and types of error measures considered here. Scrambled QMC rules have proven to be popular because of (i) their good performance in solving application problems, (ii) the explicit constructions of the generator matrices and point sets, and (iii) their strong theoretical justification. As has been noted elsewhere, $\mathcal{H}_{d, \eta, \boldsymbol{\beta}}^{\mathrm{wav}}$ is a natural space for studying rules based on $(t, m, d)$-nets because multivariate Haar wavelets with coarse structure are integrated exactly. On the other hand, the space $\mathcal{H}_{d, \gamma}^{\mathrm{SH}}$ has a natural definition based on derivatives of the integrand. Considering the randomized worst-case setting and the randomized setting allows us to derive weaker sufficient conditions for strong tractability than those that have been derived in the (deterministic) worst-case setting. This study does restrict itself to QMC algorithms when deriving necessary and sufficient conditions for tractability. The question of necessary conditions for general linear algorithms is also of interest, but it is not addressed here.

Let $\mathcal{H}_{d}$ stand for $\mathcal{H}_{d, \eta, \boldsymbol{\beta}}^{\mathrm{wav}}$ or $\mathcal{H}_{d, \boldsymbol{\gamma}}^{\mathrm{SH}}$, and let $K_{d}$ be the reproducing kernel for $\mathcal{H}_{d}$. Suppose that

$$
P_{n}^{\mathrm{sc}}=\left\{\mathbf{x}_{1}, \ldots, \mathbf{x}_{n}\right\}
$$

is a scrambled version of the original point set

$$
P_{n}=\left\{\mathbf{a}_{1}, \ldots, \mathbf{a}_{n}\right\}
$$

according to the scrambling scheme of Owen [13. The randomized worst-case error of a scrambled QMC quadrature rule $Q_{n, d}$ over the unit ball of $\mathcal{H}_{d}$ is defined as

$$
e^{\mathrm{w}}\left(P_{n}, \mathcal{H}_{d}\right)=\left(E\left[\sup _{f \in \mathcal{H}_{d},\|f\| \leq 1} \mid I_{d}(f)-Q_{n, d}\left(f, P_{n}^{\mathrm{sc}}\right)\right]^{2}\right)^{1 / 2} .
$$


The randomized error of a scrambled QMC quadrature rule $Q_{n, d}$ over the unit ball of $\mathcal{H}_{d}$ is defined as

$$
e^{\mathrm{r}}\left(P_{n}, \mathcal{H}_{d}\right)=\left(\sup _{f \in \mathcal{H}_{d},\|f\| \leq 1} E\left|I_{d}(f)-Q_{n, d}\left(f, P_{n}^{\mathrm{sc}}\right)\right|^{2}\right)^{1 / 2} .
$$

Here $\|\cdot\|$ denotes the norm in $\mathcal{H}_{d}$, and the expectation $E$ is taken with respect to scrambled sample points $\mathbf{x}_{i}$. For $n=0$ we set $Q_{n, d}=0$ so that the initial errors are

$$
e^{\mathrm{w}}\left(\emptyset, \mathcal{H}_{d}\right)=e^{\mathrm{r}}\left(\emptyset, \mathcal{H}_{d}\right)=\sup _{f \in \mathcal{H}_{d},\|f\| \leq 1}\left|I_{d}(f)\right| .
$$

Note that $e^{\mathrm{w}}\left(P_{n}, \mathcal{H}_{d}\right) \geq e^{\mathrm{r}}\left(P_{n}, \mathcal{H}_{d}\right)$ for any $P_{n}$ and $\mathcal{H}_{d}$.

For any $\varepsilon \in(0,1)$ and $d \geq 1$, let $n^{*}(\varepsilon, d)$ denote the smallest number of points $n$ required by the best $\mathrm{QMC}$ rule, $Q_{n, d}$, to ensure that the minimal $e^{*}\left(P_{n}, \mathcal{H}_{d}\right)$ is no larger than $\varepsilon e^{*}\left(\emptyset, \mathcal{H}_{d}\right)$. Here $*$ represents either w or $\mathrm{r}$. QMC strong tractability means that $n^{*}(\varepsilon, d) \leq C \varepsilon^{-p}$ for some finite $p$, called the exponent of QMC strong tractability, and for some constant $C$ independent of $d$.

The main results of this article are Theorems 1,4, which provide necessary and sufficient conditions on strong tractability for the spaces $\mathcal{H}_{d, \eta, \boldsymbol{\beta}}^{\text {wav }}$ and $\mathcal{H}_{d, \boldsymbol{\gamma}}^{\mathrm{SH}}$ in the randomized worst-case setting and randomized setting. These results are summarized in the Table 1. The quadrature rules are all based on scrambled Niederreiter nets or sequences in prime power base $b$. Net rules use the first $n=b^{m}$ points of the sequence for integer $m$. The asymptotic orders of the quadrature errors are given under the assumption that the sufficient condition for strong tractability holds. The parameter $\delta$ is an arbitrary positive number, and the notation $(x)_{+}$ means $\max (x, 0)$.

TABLE 1. Summary results from Theorems 14

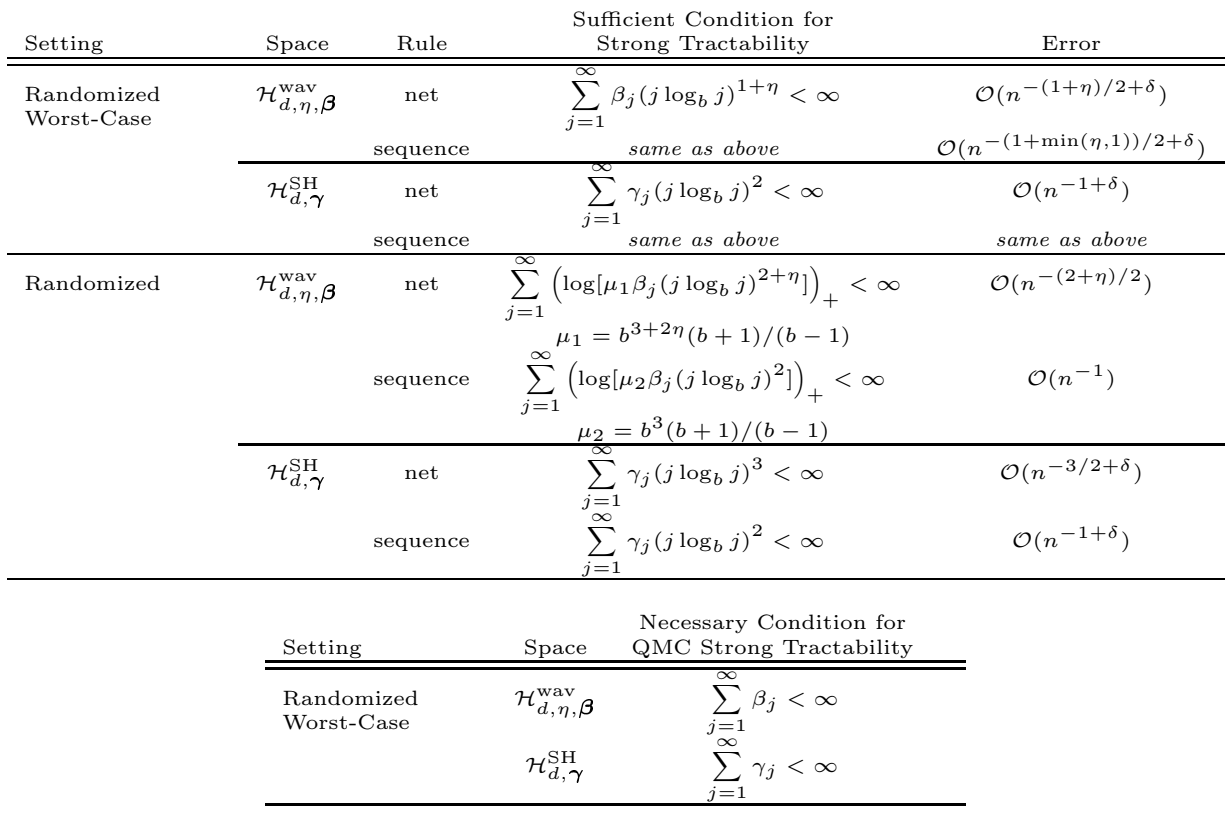


There are a few points worth noting about these results:

(i) For the space $\mathcal{H}_{d, \eta, \boldsymbol{\beta}}^{\text {wav }}$ the strong tractability conditions in the randomized worst-case setting are stronger than those in the randomized case setting.

(ii) In the randomized worst-case setting the condition for strong tractability and the order of the error are the same for $\mathcal{H}_{d, \gamma}^{\mathrm{SH}}$ as for $\mathcal{H}_{d, 1, \gamma}^{\mathrm{wav}}$.

(iii) For sequence rules the order of convergence can never be better than $\mathcal{O}\left(n^{-1}\right)$, no matter which setting or space of integrands.

(iv) The necessary conditions for QMC strong tractability for the two spaces are the same. These conditions are also sufficient if one is willing to accept a convergence rate of only $\mathcal{O}\left(n^{-1 / 2}\right)([17$, [19, 25]).

Before ending this section, we briefly describe some properties of the Niederreiter sequence. For details of the construction one may refer to [10, 11. Let $b$ be a prime power base, and let $p_{1}, p_{2}, \ldots, p_{d}$ be the first $d$ monic irreducible polynomials over the finite field $F_{b}$ according to nondecreasing degree. For a Niederreiter $(t, d)$ sequence in base $b$, the value of $t \geq 0$ is given by

$$
t=\sum_{j=1}^{d}\left[\operatorname{deg}\left(p_{j}\right)-1\right]
$$

This parameter denotes the quality of a sequence, with smaller numbers being better. The Niederreiter sequence has the following telescopic property: In order to obtain a sequence in dimension $d>1$, it suffices to add the last component $a_{i d}$ to the term of the $d-1$ dimensional sequence $\left(a_{i 1}, \ldots, a_{i, d-1}\right)$, i.e., with the previous $d-1$ components kept unchanged. Furthermore, the $j$-th component of the sequence depends on the $j$-th monic irreducible polynomial regardless of the total dimension $d$. This property allows us to investigate the quality of any lower dimensional projections of the sequence. Let $P_{n}$ be the Niederreiter $(t, m, d)$-net with $n=b^{m}$ or the first $n$ points of the Niederreiter $(t, d)$-sequence in prime power base $b$. For any nonempty subset $u$ of the coordinate axes $\mathcal{A}=\{1, \ldots, d\}$, let $P_{n, u}$ be the projection of $P_{n}$ onto the $|u|$-dimensional unit cube $[0,1)^{u}$, where $|u|$ denotes the cardinality of $u$. Then $P_{n, u}$ forms a $\left(t_{u},|u|\right)$-sequence in base $b$ with

$$
t_{u}=\sum_{j \in u}\left[\operatorname{deg}\left(p_{j}\right)-1\right] .
$$

To denote the different $t$-values for different projections of the net or sequence, one may write $\left(\left(t_{u}\right), m, d\right)$-net and $\left(\left(t_{u}\right), d\right)$-sequence as done by Hickernell and Yue 8$]$, where $\left(t_{u}\right)$ is a $2^{d}-1$ dimensional vector. An upper bound on $\operatorname{deg}\left(p_{j}\right)$ in (3) is given as follows ([1], 23]):

Lemma 1. For any prime power base b,

$$
\operatorname{deg}\left(p_{j}\right)<\log _{b} j+\log _{b} \log _{b}(j+b)+2, \quad j=1,2, \ldots
$$

\section{Integration OVER $\mathcal{H}_{d, \eta, \boldsymbol{\beta}}^{\text {wav }}$ IN THE RANDOMized WORST-CASE SETting}

In this section we first briefly review base $b$ scrambling proposed by Owen [13]. Then we consider the QMC strong tractability problem of multivariate integration using scrambled Niederreiter points in the randomized worst-case setting over the weighted Hilbert space of Haar-like wavelets, $\mathcal{H}_{d, \eta, \boldsymbol{\beta}}^{\mathrm{wav}}$. 
2.1. Base $b$ scrambling. The base $b$ scrambling proposed by Owen 13 can be briefly described as follows. Consider the set $P_{n}=\left\{\mathbf{a}_{1}, \ldots, \mathbf{a}_{n}\right\}$ and write the components of $\mathbf{a}_{i}$ as $a_{i j}=\sum_{k=1}^{\infty} a_{i j k} b^{-k}$. For $i=1, \ldots, n$, let $\mathbf{x}_{i}=\left(x_{i 1}, \ldots, x_{i d}\right)$ with $x_{i j}=\sum_{k=1}^{\infty} x_{i j k} b^{-k}$, where $x_{i j k}$ is a random permutation applied to $a_{i j k}$ but that depends on $a_{i j h}$ for $h=1, \ldots, k$. The $\mathbf{x}_{i}$ 's satisfy the following rules:

(1) Each digit $x_{i j k}$ is uniformly distributed on the set $\{0,1, \ldots, b-1\}$.

(2) For any two points $\mathbf{x}_{i}$ and $\mathbf{x}_{i^{\prime}}$ the $s$ pairs $\left(x_{i 1}, x_{i^{\prime} 1}\right), \ldots,\left(x_{i d}, x_{i^{\prime} d}\right)$ are mutually independent.

(3) If $a_{i j}$ and $a_{i^{\prime} j}$ share the same first $k$ digits but their $k+1^{\text {st }}$ digits are different, then

(a) $x_{i j h}=x_{i^{\prime} j h}$ for $h=1, \ldots, k$;

(b) the pair $\left(x_{i j k+1}, x_{i^{\prime} j k+1}\right)$ is uniformly distributed on the set $\{0,1, \ldots$, $b-1\}^{2} \backslash\{(0,0),(1,1) \ldots,(b-1, b-1)\} ;$ and

(c) $x_{i j k+2}, x_{i j k+3}, \cdots, x_{i^{\prime} j k+2}, x_{i^{\prime} j k+3}, \cdots$ are mutually independent.

We call this a base $b$ scrambling scheme and we call the sequence $P_{n}^{\text {sc }}=\left\{\mathbf{x}_{1}, \ldots, \mathbf{x}_{n}\right\}$ a scrambled version of $P_{n}=\left\{\mathbf{a}_{1}, \ldots, \mathbf{a}_{n}\right\}$.

The following geometrical description of this scheme may help us visualize the randomization. Begin by partitioning the unit cube $[0,1)^{d}$ along the $x_{1}$ axis into $b$ parallel $b$-boxes of the form $[\ell / b,(\ell+1) / b) \times[0,1)^{d-1}$ for $\ell=0, \ldots, b-1$. Then randomly permute those $b$-boxes replacing them in one of the $b$ ! possible orders, each such order having probability $1 / b$ !. Next take each such $b$-box in turn, partition it into $b$ congruent $b$-boxes of volume $b^{-2}$ along the $x_{1}$ axis, and randomly permute those $b$-boxes. Then repeat this process on the $b^{2} b$-boxes of volume $b^{-2}$, then on the $b^{3} b$-boxes of volume $b^{-3}$ and so on, ad infinitum. In practice this can stop when the $b$-boxes are narrow compared to machine precision. The full scrambling involves applying the above operations along the other $d-1$ axes $x_{2}, \cdots, x_{d}$ as well. All of the many permutations used are assumed to be statistically independent.

2.2. The definition of the space $\mathcal{H}_{d, \eta, \boldsymbol{\beta}}^{\text {wav }}$. For a given base $b$, define the univariate basic wavelet functions

$$
\psi_{c}(x)=b^{1 / 2} 1_{\lfloor b x\rfloor=c}-b^{-1 / 2} 1_{\lfloor x\rfloor=0}, \quad x \in[0,1), \quad c=0,1, \ldots, b-1,
$$

where $1_{\{\cdot\}}$ denotes the characteristic function and $\lfloor x\rfloor$ denotes the floor function of $x$ or the greatest integer less than or equal to $x$. For integers $\kappa \geq 0$ and $0 \leq \tau<b^{\kappa}$ the dilated and translated versions of the above functions are the following univariate wavelets:

$$
\psi_{\kappa \tau c}(x)=b^{\kappa / 2} \psi_{c}\left(b^{\kappa} x-\tau\right)=b^{(\kappa-1) / 2}\left(b 1_{\left\lfloor b^{\kappa+1} x\right\rfloor=b \tau+c}-1_{\left\lfloor b^{\kappa} x\right\rfloor=\tau}\right) .
$$

In the multidimensional case, for any nonempty subset $u$ of $\mathcal{A}=\{1, \ldots, d\}$, let $\boldsymbol{\kappa}$ denote a $|u|$-vector with integer components $\kappa_{j} \in\{0,1,2, \ldots\}$ for $j \in u$, let $\boldsymbol{\tau}$ denote a $|u|$-vector with integer components $\tau_{j} \in\left\{0,1, \ldots, b^{\kappa_{j}}-1\right\}$ for $j \in u$, and let $\mathbf{c}$ denote a $|u|$-vector with integer components $c_{j} \in\{0,1, \ldots, b-1\}$ for $j \in u$. Let $\psi_{u \kappa \tau \mathbf{c}}$ be a product over $j \in u$ of the dilated and translated wavelets, i.e.,

$$
\psi_{u \kappa \tau \mathbf{c}}(\mathbf{x}) \equiv \prod_{j \in u} \psi_{\kappa_{j} \tau_{j} c_{j}}\left(x_{j}\right)=b^{\left(\|\boldsymbol{\kappa}\|_{1}-|u|\right) / 2} \prod_{j \in u}\left(b 1_{\left\lfloor b^{\kappa_{j}+1} x_{j}\right\rfloor=b \tau_{j}+c_{j}}-1_{\left\lfloor b^{\kappa_{j}} x_{j}\right\rfloor=\tau_{j}}\right),
$$

where $\|\boldsymbol{\kappa}\|_{1}=\sum_{j \in u} \kappa_{j}$. For $u=\emptyset$ we take by convention $\psi_{u \kappa \tau \mathbf{c}}(\mathbf{x})=\psi_{\emptyset}(\mathbf{x})=1$. The wavelets defined above are not orthogonal nor linearly independent, but they 
are nearly so. As observed in [14,

$$
\begin{gathered}
\sum_{c_{j}=0}^{b-1} \psi_{u \kappa \boldsymbol{\tau} \mathbf{c}}(\mathbf{x})=0, \\
\forall u \neq \emptyset, \forall \boldsymbol{\kappa}, \boldsymbol{\tau}, \forall j \in u, \forall c_{j^{\prime}} \in\{0,1, \cdots, b-1\} \text { with } j \neq j^{\prime} \in u, \\
\int_{[0,1)^{d}} \psi_{u \kappa \tau \mathbf{c}}(\mathbf{x}) \psi_{u^{\prime} \boldsymbol{\kappa}^{\prime} \boldsymbol{\tau}^{\prime} \mathbf{c}^{\prime}}(\mathbf{x}) d \mathbf{x}=1_{u=u^{\prime}} 1_{\boldsymbol{\kappa}=\boldsymbol{\kappa}^{\prime}} 1_{\boldsymbol{\tau}=\boldsymbol{\tau}^{\prime}} \prod_{j \in u}\left(1_{c_{j}=c_{j}^{\prime}}-b^{-1}\right) .
\end{gathered}
$$

Owen [14] showed that any function $f \in \mathcal{L}_{2}\left([0,1)^{d}\right)$ can be expanded in a series representation as one would expect for an orthogonal basis:

$$
f(\mathbf{x})=\sum_{u, \kappa, \tau, \mathbf{c}} \hat{f}_{u \kappa \tau \mathbf{c}} \psi_{u \kappa \tau \mathbf{c}}(\mathbf{x}),
$$

where the coefficients are given by

$$
\hat{f}_{u \kappa \tau \mathbf{c}}=\int_{[0,1)^{d}} f(\mathbf{x}) \psi_{u \kappa \tau \mathbf{c}}(\mathbf{x}) d \mathbf{x} .
$$

Now we can define our weighted space $\mathcal{H}_{d, \eta, \boldsymbol{\beta}}^{\text {wav }}$ considered in this article. Let $\boldsymbol{\beta}=\left(\beta_{1}, \ldots, \beta_{d}\right)$ be a sequence such that

$$
\beta_{1} \geq \beta_{2} \geq \cdots \geq \beta_{d}>0,
$$

and let $\beta_{\emptyset}=1$ and $\beta_{u}=\prod_{j \in u} \beta_{j}$ for any nonempty subset $u \subseteq \mathcal{A}$. Let $\omega_{u \kappa}$ be defined by

$$
\omega_{u \kappa}=\beta_{u} b^{-(1+\eta)\|\kappa\|_{1}}, \quad \text { where } \quad \eta>0 .
$$

Then define the scaled wavelets as

$$
\psi_{u \kappa \tau \mathbf{c}}^{\omega}(\mathbf{x})=\omega_{u \kappa}^{1 / 2} \psi_{u \kappa \tau \mathbf{c}}(\mathbf{x}) .
$$

As seen in Theorems 1 and 3, the value of $\eta$ controls the asymptotic rate of decay of the randomized worst-case error and the randomized error. The space $\mathcal{H}_{d, \eta, \boldsymbol{\beta}}^{\text {wav }}$ is defined as

$$
\begin{array}{r}
\mathcal{H}_{d, \eta, \boldsymbol{\beta}}^{\mathrm{wav}}=\left\{f(\mathbf{x})=\sum_{u, \boldsymbol{\kappa}, \boldsymbol{\tau}, \mathbf{c}} \hat{f}_{u \kappa \tau \mathbf{c}}^{\omega} \psi_{u \kappa \tau \mathbf{c}}^{\omega}(\mathbf{x}): \sum_{u, \boldsymbol{\kappa}, \boldsymbol{\tau}, \mathbf{c}}\left(\hat{f}_{u \kappa \tau \mathbf{c}}^{\omega}\right)^{2}<\infty,\right. \\
\left.\sum_{c_{j}=0}^{b-1} \hat{f}_{u \kappa \tau \mathbf{c}}^{\omega}=0, \forall j \in u, \forall u, \boldsymbol{\kappa}, \boldsymbol{\tau}, \mathbf{c}_{u-\{j\}}\right\} .
\end{array}
$$

Because the wavelets are not linearly independent, the condition on the sum of the series coefficients is required to insure that the series expression for $f \in \mathcal{H}_{d, \eta, \boldsymbol{\beta}}^{\text {wav }}$ is unique.

The inner product in $\mathcal{H}_{d, \eta, \boldsymbol{\beta}}^{\text {wav }}$ is defined as

$$
\langle f, g\rangle_{\mathrm{wav}, d, \eta, \boldsymbol{\beta}}=\sum_{u, \kappa, \tau, \mathbf{c}} \hat{f}_{u \kappa \tau \mathbf{c}}^{\omega} \hat{g}_{u \kappa \tau \mathbf{c}}^{\omega}
$$

for

$$
f(\mathbf{x})=\sum_{u, \kappa, \boldsymbol{\tau}, \mathbf{c}} \hat{f}_{u \kappa \tau \mathbf{c}}^{\omega} \psi_{u \kappa \tau \mathbf{c}}^{\omega}(\mathbf{x}) \text { and } g(\mathbf{x})=\sum_{u, \boldsymbol{\kappa}, \boldsymbol{\tau}, \mathbf{c}} \hat{g}_{u \kappa \tau \mathbf{c}}^{\omega} \psi_{u \kappa \tau \mathbf{c}}^{\omega}(\mathbf{x}),
$$


and the squared norm is

(10)

$$
\|f\|_{\mathrm{wav}, d, \eta, \boldsymbol{\beta}}^{2}=\sum_{u, \boldsymbol{\kappa}, \boldsymbol{\tau}, \mathbf{c}}\left(\hat{f}_{u \kappa \tau \mathbf{c}}^{\omega}\right)^{2}=\sum_{u, \boldsymbol{\kappa}, \boldsymbol{\tau}, \mathbf{c}} \omega_{u \kappa}^{-1} \hat{f}_{u \kappa \tau \mathbf{c}}^{2}=\sum_{u, \boldsymbol{\kappa}, \boldsymbol{\tau}, \mathbf{c}} \beta_{u}^{-1} b^{2(1+\eta)\|\boldsymbol{\kappa}\|_{1}} \hat{f}_{u \kappa \tau \mathbf{c}}^{2},
$$

where $\hat{f}_{u \kappa \tau \mathbf{c}}$ is as defined in (77). It can be verified that the space $\mathcal{H}_{d, \eta, \boldsymbol{\beta}}^{\text {wav }}$ is a reproducing kernel Hilbert space, and its reproducing kernel is

$$
K_{d, \eta, \boldsymbol{\beta}}^{\mathrm{sc}}(\mathbf{x}, \mathbf{y})=\sum_{u, \boldsymbol{\kappa}, \boldsymbol{\tau}, \mathbf{c}} \omega_{u \kappa} \psi_{u \kappa \tau \mathbf{c}}(\mathbf{x}) \psi_{u \kappa \tau \mathbf{c}}(\mathbf{y}) .
$$

Reproducing kernels of the form (11) have the property that their values do not change under scrambling. Such kernels are said to be scramble-invariant. In other words, if $\mathbf{x}_{i}$ and $\mathbf{x}_{i^{\prime}}$ are the scrambled versions of two points, $\mathbf{a}_{i}$ and $\mathbf{a}_{i^{\prime}}$ in $P_{n}=\left\{\mathbf{a}_{1}, \ldots, \mathbf{a}_{n}\right\} \subset[0,1)^{d}$, then $K_{d, \eta, \boldsymbol{\beta}}^{\mathrm{sc}}\left(\mathbf{x}_{i}, \mathbf{x}_{i^{\prime}}\right)=K_{d, \eta, \boldsymbol{\beta}}^{\mathrm{sc}}\left(\mathbf{a}_{i}, \mathbf{a}_{i^{\prime}}\right)$ with probability one ([8]). The randomized worst-case error defined in (11) for $\mathcal{H}_{d, \eta, \boldsymbol{\beta}}^{\text {wav }}$ is

$$
e^{\mathrm{w}}\left(P_{n}, \mathcal{H}_{d, \eta, \boldsymbol{\beta}}^{\mathrm{wav}}\right)=\left(E\left[\sup _{\|f\|_{\text {wav }, d, \eta, \boldsymbol{\beta}} \leq 1}\left|I_{d}(f)-Q_{n, d}\left(f, P_{n}^{\mathrm{sc}}\right)\right|\right]^{2}\right)^{1 / 2} .
$$

It is known that $e^{\mathrm{w}}\left(P_{n}, \mathcal{H}_{d, \eta, \boldsymbol{\beta}}^{\mathrm{wav}}\right)$ is the discrepancy of $P_{n}$ based on the kernel $K_{d, \eta, \boldsymbol{\beta}}^{\mathrm{sc}}$, i.e.,

$$
\begin{aligned}
{\left[e^{\mathrm{w}}\left(P_{n}, \mathcal{H}_{d, \eta, \boldsymbol{\beta}}^{\mathrm{wav}}\right)\right]^{2}=} & \int_{[0,1)^{2 d}} K_{d, \eta, \boldsymbol{\beta}}^{\mathrm{sc}}(\mathbf{x}, \mathbf{y}) d \mathbf{x} d \mathbf{y} \\
& -\frac{2}{n} \sum_{i=1}^{n} \int_{[0,1)^{d}} K_{d, \eta, \boldsymbol{\beta}}^{\mathrm{sc}}\left(\mathbf{a}_{i}, \mathbf{y}\right) d \mathbf{y}+\frac{1}{n^{2}} \sum_{i=1}^{n} \sum_{i^{\prime}=1}^{n} K_{d, \eta, \boldsymbol{\beta}}^{\mathrm{sc}}\left(\mathbf{a}_{i}, \mathbf{a}_{i^{\prime}}\right) .
\end{aligned}
$$

For $n \geq 1$, from 8 we have

$$
\begin{aligned}
{\left[e^{\mathrm{w}}\left(P_{n}, \mathcal{H}_{d, \eta, \boldsymbol{\beta}}^{\mathrm{wav}}\right)\right]^{2}=} & \frac{1}{n^{2}} \sum_{i=1}^{n} \sum_{i^{\prime}=1}^{n} \sum_{u \neq \emptyset} \sum_{\kappa} \beta_{u} b^{-\eta\|\boldsymbol{\kappa}\|_{1}} \\
& \times \prod_{j \in u}\left[b N_{\kappa_{j}}\left(a_{i j}, a_{i^{\prime} j}\right)-W_{\kappa_{j}}\left(a_{i j}, a_{i^{\prime} j}\right)\right],
\end{aligned}
$$

where $N_{k}(x, y)$ and $W_{k}(x, y)$ are defined as

$$
N_{k}(x, y)=1_{\left\lfloor b^{k+1} x\right\rfloor=\left\lfloor b^{k+1} y\right\rfloor}, \quad W_{k}(x, y)=1_{\left\lfloor b^{k} x\right\rfloor=\left\lfloor b^{k} y\right\rfloor} .
$$

The "narrow" function, $N_{k}(x, y)$, measures whether more than the first $k$ digits of $x$ and $y$ are the same. The "wide" function, $W_{k}(x, y)$, measures whether at least the first $k$ digits of $x$ and $y$ are the same. By defining

$$
\Gamma_{u \kappa}\left(P_{n}\right)=\frac{1}{n(b-1)^{|u|}} \sum_{i=1}^{n} \sum_{i^{\prime}=1}^{n} \prod_{j \in u}\left[b N_{\kappa_{j}}\left(a_{i j}, a_{i^{\prime} j}\right)-W_{\kappa_{j}}\left(a_{i j}, a_{i^{\prime} j}\right)\right],
$$

(14) can be rewritten as

$$
\left[e^{\mathrm{w}}\left(P_{n}, \mathcal{H}_{d, \eta, \boldsymbol{\beta}}^{\mathrm{wav}}\right)\right]^{2}=\frac{1}{n} \sum_{u \neq \emptyset} \beta_{u}(b-1)^{|u|} \sum_{\boldsymbol{\kappa}} b^{-\eta\|\boldsymbol{\kappa}\|_{1}} \Gamma_{u \boldsymbol{\kappa}}\left(P_{n}\right) .
$$

The $\Gamma_{u \kappa}\left(P_{n}\right)$ are called gain coefficients under scrambling for the point set $P_{n}([14])$. 
2.3. Upper bounds on $e^{\mathrm{w}}\left(P_{n}, \mathcal{H}_{d, \eta, \boldsymbol{\beta}}^{\mathrm{wav}}\right)$. The following lemma gives upper bounds on the $\Gamma_{u \kappa}$ for nets and sequences in base $b$. See [16] and 8] for more details on the gain coefficients.

Lemma 2. Let $P_{n}^{\text {net }}$ be a $\left(\left(t_{u}\right), m, d\right)$-net in base $b$, and let $P_{n}^{\text {seq }}$ be the first $n$ points of a $\left(\left(t_{u}\right), d\right)$-sequence in base $b$. Then

$$
\Gamma_{u \boldsymbol{\kappa}}\left(P_{n}^{\text {net }}\right)\left\{\begin{array}{l}
=0, \quad\|\boldsymbol{\kappa}\|_{1} \leq m-t_{u}-|u|, \\
\leq b^{t_{u}}\left(\frac{b+1}{b-1}\right)^{|u|}, \quad\|\boldsymbol{\kappa}\|_{1}>m-t_{u}-|u|
\end{array}\right.
$$

and

$$
\Gamma_{u \kappa}\left(P_{n}^{\mathrm{seq}}\right) \leq \frac{1}{n} b^{t_{u}+\min \left(q+1, t_{u}+|u|+\|\kappa\|_{1}\right)}\left(\frac{b+1}{b-1}\right)^{|u|+1},
$$

where $q$ is the largest integer such that $b^{q} \leq n$.

Some improvements on the upper bounds on $\Gamma_{u \kappa}$ can be found in [12]. From Lemma 2 we can obtain an upper bound on the inner sum over $\kappa$ in (16).

Lemma 3. Let $P_{n}^{\text {net }}$ and $P_{n}^{\text {seq }}$ be defined as in Lemma 2. Then for any nonempty subset $u \subseteq \mathcal{A}$, we have

$$
\begin{aligned}
& \sum_{\kappa} b^{-\eta\|\kappa\|_{1}} \Gamma_{u \kappa}\left(P_{n}^{\text {net }}\right) \leq C_{1} n^{-\eta}(\log n)^{|u|} \theta^{|u|} b^{(1+\eta) t_{u}}, \\
& \sum_{\kappa} b^{-\eta\|\kappa\|_{1}} \Gamma_{u \kappa}\left(P_{n}^{\mathrm{seq}}\right) \leq C_{2} n^{-\bar{\eta}}(\log n)^{|u|} \theta^{|u|} b^{(1+\bar{\eta}) t_{u}},
\end{aligned}
$$

where $\bar{\eta}=\min (\eta, 1), \theta=\nu b^{\eta}(b+1) /[(b-1) \log b], \nu=\max \left(1,1 /\left(b^{\eta}-1\right)\right)$, and

$$
C_{1}=\left\{\begin{array}{cl}
1, & \text { if } b^{\eta}=2, \\
\frac{1}{\left|2-b^{\eta}\right|}, & \text { otherwise, }
\end{array} \quad C_{2}=\frac{b+1}{b-1}\left(A+b C_{1}\right),\right.
$$

where

$$
A=\left\{\begin{array}{cc}
1, & \text { if } \eta=1, \\
\frac{b^{1-\bar{\eta}}}{\left|1-b^{\eta}\right|}, & \text { otherwise. }
\end{array}\right.
$$

Proof. For any nonempty subset $u$ and $|u|$-vector $\boldsymbol{\kappa}$ put $r=|u|$ and $k=\|\boldsymbol{\kappa}\|_{1}$ for simplicity. For the set $P_{n}^{\text {net }}$, from (17) we have

$$
\begin{aligned}
\sum_{\kappa} b^{-\eta\|\boldsymbol{\kappa}\|_{1}} \Gamma_{u \kappa}\left(P_{n}^{\text {net }}\right) & \leq b^{t_{u}}\left(\frac{b+1}{b-1}\right)^{r} \sum_{\kappa:\|\boldsymbol{\kappa}\|_{1} \geq m-t_{u}-r+1} b^{-\eta\|\boldsymbol{\kappa}\|_{1}} \\
& =b^{t_{u}}\left(\frac{b+1}{b-1}\right)^{r} \sum_{k=m-t_{u}-r+1}^{\infty}\left(\begin{array}{c}
r-1+k \\
r-1
\end{array}\right) b^{-\eta k} .
\end{aligned}
$$

Replacing $k$ with $m-t_{u}-r+1+\ell$ in (23) and applying the identity

$$
\left(\begin{array}{c}
m-t_{u}+\ell \\
r-1
\end{array}\right)=\sum_{\alpha=0}^{r-1}\left(\begin{array}{c}
m-t_{u} \\
\alpha
\end{array}\right)\left(\begin{array}{c}
\ell \\
r-1-\alpha
\end{array}\right)
$$


we can rewrite the last sum in (23) as

$$
\begin{aligned}
& \sum_{k=m-t_{u}-r+1}^{\infty}\left(\begin{array}{c}
r-1+k \\
r-1
\end{array}\right) b^{-\eta k} \\
& =b^{-\eta\left(m-t_{u}-r+1\right)} \sum_{\ell=0}^{\infty}\left(\begin{array}{c}
m-t_{u}+\ell \\
r-1
\end{array}\right) b^{-\eta \ell} \\
& =b^{-\eta\left(m-t_{u}-r+1\right)} \sum_{\ell=0}^{\infty} \sum_{\alpha=0}^{r-1}\left(\begin{array}{c}
m-t_{u} \\
\alpha
\end{array}\right)\left(\begin{array}{c}
\ell \\
r-1-\alpha
\end{array}\right) b^{-\eta \ell} \\
& =b^{-\eta\left(m-t_{u}-r+1\right)} \sum_{\alpha=0}^{r-1}\left(\begin{array}{c}
m-t_{u} \\
\alpha
\end{array}\right) \sum_{\ell=r-1-\alpha}^{\infty}\left(\begin{array}{c}
\ell \\
r-1-\alpha
\end{array}\right) b^{-\eta \ell} .
\end{aligned}
$$

Replacing $\ell$ with $r-1-\alpha+j$ in the inner sum gives

$$
\begin{aligned}
& \sum_{\ell=r-1-\alpha}^{\infty}\left(\begin{array}{c}
\ell \\
r-1-\alpha
\end{array}\right) b^{-\eta \ell}=b^{-\eta(r-1-\alpha)} \sum_{j=0}^{\infty}\left(\begin{array}{c}
r-1-\alpha+j \\
r-1-\alpha
\end{array}\right) b^{-\eta j} \\
& =b^{-\eta(r-1-\alpha)}\left(1-b^{-\eta}\right)^{-(r-\alpha)}=b^{\eta}\left(b^{\eta}-1\right)^{\alpha-r}
\end{aligned}
$$

where the second equality holds due to the identity

$$
(1-x)^{-a}=\sum_{j=0}^{\infty}\left(\begin{array}{c}
a-1+j \\
a-1
\end{array}\right) x^{j}, \quad a>0, \quad|x|<1 .
$$

Inserting (25) into (24) leads to

$$
\sum_{k=m-t_{u}-r+1}^{\infty}\left(\begin{array}{c}
r-1+k \\
r-1
\end{array}\right) b^{-\eta k}=b^{-\eta\left(m-t_{u}-r\right)} \sum_{\alpha=0}^{r-1}\left(\begin{array}{c}
m-t_{u} \\
\alpha
\end{array}\right)\left(b^{\eta}-1\right)^{\alpha-r}
$$

Note that for $0 \leq \alpha \leq r-1$ we have $\left(\begin{array}{c}m-t_{u} \\ \alpha\end{array}\right) \leq m^{r-1}<m^{r}$, and

$$
\sum_{\alpha=0}^{r-1}\left(b^{\eta}-1\right)^{\alpha-r}=\left\{\begin{array}{cl}
r, & \text { if } b^{\eta}=2, \\
\frac{\left(b^{\eta}-1\right)^{-r}-1}{2-b^{\eta}}, & \text { otherwise, }
\end{array} \leq\left\{\begin{array}{cl}
m, & \text { if } b^{\eta}=2 \\
\frac{\nu^{r}}{\left|2-b^{\eta}\right|}, & \text { otherwise }
\end{array}\right.\right.
$$

where $\nu=\max \left(1,1 /\left(b^{\eta}-1\right)\right)$. These upper bounds, together with the equality in (26) yields

$$
\sum_{k=m-t_{u}-r+1}^{\infty}\left(\begin{array}{c}
r-1+k \\
r-1
\end{array}\right) b^{-\eta k} \leq C_{1} m^{r} \nu^{r} b^{-\eta\left(m-t_{u}-r\right)},
$$

where $C_{1}$ is as shown in (21). From (23) and (27), and noting that $n=b^{m}$ and $m=\log n / \log b$ for the net in base $b$, we have

$$
\begin{aligned}
\sum_{\kappa} b^{-\eta\|\kappa\|_{1}} \Gamma_{u \kappa}\left(P_{n}^{\text {net }}\right) & \leq C_{1} m^{r} b^{-\eta m}\left(\frac{\nu b^{\eta}(b+1)}{b-1}\right)^{r} b^{(1+\eta) t_{u}} \\
& \leq C_{1} n^{-\eta}(\log n)^{r} \theta^{r} b^{(1+\eta) t_{u}}
\end{aligned}
$$

where $\theta=\nu b^{\eta}(b+1) /[(b-1) \log b]$, as shown in the lemma. This is the inequality in (19). 
For the set $P_{n}^{\text {seq }}$, from (18) we have

$$
\begin{aligned}
& \sum_{\kappa} b^{-\eta\|\boldsymbol{\kappa}\|_{1}} \Gamma_{u \kappa}\left(P_{n}^{\mathrm{seq}}\right) \\
& \quad \leq n^{-1} b^{t_{u}}\left(\frac{b+1}{b-1}\right)^{r+1} \sum_{k=1}^{\infty}\left(\begin{array}{c}
r-1+k \\
r-1
\end{array}\right) b^{\min \left(q+1, t_{u}+r+k\right)-\eta k} .
\end{aligned}
$$

The sum above is split into two parts. Noting that $\left(\begin{array}{c}r-1+k \\ r-1\end{array}\right)<\left(\begin{array}{c}q \\ r-1\end{array}\right)<q^{r-1}$ for $k \leq q-t_{u}-r$, the first part is

$$
\begin{aligned}
& \sum_{k=0}^{q-t_{u}-r}\left(\begin{array}{c}
r-1+k \\
r-1
\end{array}\right) b^{t_{u}+r+k-\eta k} \\
& \quad \leq q^{r-1} \sum_{k=0}^{q-t_{u}-r} b^{t_{u}+r+(1-\eta) k} \\
& \quad=q^{r-1} b^{t_{u}+r} \times\left\{\begin{array}{cc}
\frac{1-b^{(1-\eta)\left(q-t_{u}-r+1\right)}}{1-b^{1-\eta}}, & \eta \neq 1, \\
q-t_{u}-r+1, & \eta=1,
\end{array}\right. \\
& \quad \leq A q^{r} b^{(1-\bar{\eta}) q+\bar{\eta}\left(t_{u}+r\right),}
\end{aligned}
$$

where $\bar{\eta}=\min (\eta, 1)$ and $A$ is as shown in (22).

The second part is similar to the sum for the net above. Compared with (27) we have

$$
\sum_{k=q-t_{u}-r+1}^{\infty}\left(\begin{array}{c}
r-1+k \\
r-1
\end{array}\right) b^{q+1-\eta k} \leq C_{1} q^{r} \nu^{r} b^{(1-\eta) q+\eta\left(t_{u}+r\right)+1}, \quad \eta>0,
$$

where $C_{1}$ is as shown in (21).

From (28)- (30) and noting that $\bar{\eta} \leq \eta, 1 \leq \nu, b^{q} \leq n$ and $q \leq \log n / \log b$, we have

$$
\begin{aligned}
\sum_{\kappa} b^{-\eta\|\kappa\|_{1}} \Gamma_{u \kappa}\left(P_{n}^{\mathrm{seq}}\right) \\
\quad \leq n^{-1} b^{t_{u}}\left(\frac{b+1}{b-1}\right)^{r+1} q^{r}\left(A b^{(1-\bar{\eta}) q+\bar{\eta}\left(t_{u}+r\right)}+C_{1} \nu^{r} b^{(1-\eta) q+\eta\left(t_{u}+r\right)+1}\right) \\
\quad \leq n^{-1} b^{t_{u}}\left(\frac{b+1}{b-1}\right)^{r+1} q^{r} \nu^{r} b^{(1-\bar{\eta}) q+\bar{\eta}\left(t_{u}+r\right)}\left(A+b C_{1}\right) \\
\quad \leq C_{2} n^{-\bar{\eta}}(\log n)^{r} \theta^{r} b^{(1+\eta) t_{u}},
\end{aligned}
$$

where $C_{2}$ and $\theta$ are as shown in the lemma. The proof is completed.

Note from (3) and (4) that for Niederreiter $\left(\left(t_{u}\right), d\right)$-sequence in base $b$,

$$
b^{t_{u}} \leq b^{|u|} \prod_{j \in u}\left[j \log _{b}(j+b)\right] .
$$

From (16), (19), (20) and (31) we have the following upper bounds on the randomized worst-case error for Niederreiter points. 
Lemma 4. Let $C_{1}, C_{2}, \theta$ and $\bar{\eta}$ be as defined in Lemma 3, and let $\widetilde{\theta}=b^{1+\eta}(b-1) \theta$. Let $P_{n}^{N-\text { net }}$ be a Niederreiter $\left(\left(t_{u}\right), m, d\right)$-net, and let $P_{n}^{N-\operatorname{seq}}$ be the set of the first $n$ points of a Niederreriter $\left(\left(t_{u}\right), d\right)$-sequence. Then

$$
\begin{aligned}
& {\left[e^{\mathrm{w}}\left(P_{n}^{N-\mathrm{net}}, \mathcal{H}_{d, \eta, \boldsymbol{\beta}}^{\mathrm{wav}}\right)\right]^{2} \leq C_{1} n^{-(1+\eta)} \sum_{u \neq \emptyset} \prod_{j \in u}\left\{\tilde{\theta} \beta_{j}\left[j \log _{b}(j+b)\right]^{1+\eta} \log n\right\},} \\
& {\left[e^{\mathrm{w}}\left(P_{n}^{N-\mathrm{seq}}, \mathcal{H}_{d, \eta, \boldsymbol{\beta}}^{\mathrm{wav}}\right)\right]^{2} \leq C_{2} n^{-(1+\bar{\eta})} \sum_{u \neq \emptyset} \prod_{j \in u}\left\{\tilde{\theta} \beta_{j}\left[j \log _{b}(j+b)\right]^{1+\eta} \log n\right\} .}
\end{aligned}
$$

2.4. Lower bounds on $e^{\mathrm{w}}\left(P_{n}, \mathcal{H}_{d, \eta, \boldsymbol{\beta}}^{\text {wav }}\right)$. We now present lower bounds on the randomized worst-case error of QMC quadrature for the weighted Hilbert spaces $\mathcal{H}_{d, \eta, \boldsymbol{\beta}}^{\text {wav }}$. The argument used is the same as in [18] and [9].

By letting

$$
R(x, y)=\sum_{k=0}^{\infty} b^{-\eta k}\left[b N_{k}(x, y)-W_{k}(x, y)\right], \quad(x, y) \in[0,1]^{2},
$$

the randomized worst-case error in (14) can be rewritten as

$$
\begin{aligned}
{\left[e^{\mathrm{w}}\left(P_{n}, \mathcal{H}_{d, \boldsymbol{\eta}, \boldsymbol{\beta}}^{\mathrm{wav}}\right)\right]^{2} } & =\frac{1}{n^{2}} \sum_{i=1}^{n} \sum_{i^{\prime}=1}^{n} \sum_{u \neq \emptyset} \sum_{\kappa} \beta_{u} b^{-\eta\|\boldsymbol{\kappa}\|_{1}} \prod_{j \in u} R\left(a_{i j}, a_{i^{\prime} j}\right) \\
& =-1+\frac{1}{n^{2}} \sum_{i=1}^{n} \sum_{i^{\prime}=1}^{n} \prod_{j=1}^{d}\left[1+\beta_{j} R\left(a_{i j}, a_{i^{\prime} j}\right)\right] .
\end{aligned}
$$

Note that the terms in the double sum in (35) may not be nonnegative. We introduce a positive sequence $\boldsymbol{\xi}=\left(\xi_{1}, \ldots, \xi_{d}\right)$ such that $\xi_{j} \leq \beta_{j}$ for all $j=1, \ldots, d$, and we later choose $\boldsymbol{\xi}$ so as to make the corresponding terms in the double sum nonnegative. Since $\xi_{j} \leq \beta_{j}$ for all $j$, we have

$$
\|f\|_{\text {wav }, d, \eta, \boldsymbol{\beta}}^{2} \leq\|f\|_{\text {wav }, d, \eta, \boldsymbol{\xi}}^{2}
$$

due to the definition of the norm in (10). This means that the unit ball of $\mathcal{H}_{d, \eta, \boldsymbol{\xi}}^{\text {wav }}$ is contained in the unit ball of $\mathcal{H}_{d, \eta, \boldsymbol{\beta}}^{\mathrm{wav}}$, and hence from the definition in (12) we have

$$
e^{\mathrm{w}}\left(P_{n}, \mathcal{H}_{d, \eta, \boldsymbol{\xi}}^{\mathrm{wav}}\right) \leq e^{\mathrm{w}}\left(P_{n}, \mathcal{H}_{d, \eta, \boldsymbol{\beta}}^{\mathrm{wav}}\right)
$$

Thus to obtain a lower bound on $e^{\mathrm{w}}\left(P_{n}, \mathcal{H}_{d, \eta, \boldsymbol{\beta}}^{\text {wav }}\right)$, we just need to choose an appropriate sequence $\boldsymbol{\xi}$ and then obtain a lower bound on $e^{\mathrm{w}}\left(P_{n}, \mathcal{H}_{d, \eta, \boldsymbol{\xi}}^{\mathrm{wav}}\right)$.

Let $R_{\max }$ and $R_{\min }$ denote the maximum and the minimum of $R(x, y)$ on $[0,1]^{2}$. Then

$$
R_{\max }=\sum_{k=0}^{\infty} b^{-\eta k}(b-1)=\frac{b^{\eta}(b-1)}{b^{\eta}-1} \quad \text { and } \quad R_{\min }=-1 .
$$

Define

$$
\mu=\min \left(1, \frac{1}{\sup _{j} \beta_{j}}\right)
$$

and set $\xi_{j}=\mu \beta_{j}$ for $j=1, \ldots, d$. The sequence $\boldsymbol{\xi}=\left(\xi_{1}, \ldots, \xi_{d}\right)$ defined this way is positive, and for all $j=1, \ldots, d$ we have

$$
1+\xi_{j} R(x, y)=1+\mu \beta_{j} R(x, y) \geq 1+\mu \beta_{j} R_{\min }=1-\mu \beta_{j} \geq 0 .
$$


Therefore, from (35) and (37) we have

$$
\begin{aligned}
{\left[e^{\mathrm{w}}\left(P_{n}, \mathcal{H}_{d, \eta, \boldsymbol{\xi}}^{\mathrm{wav}}\right)\right]^{2} } & \geq-1+\frac{1}{n^{2}} \sum_{i=1}^{n} \prod_{j=1}^{d}\left[1+\xi_{j} R\left(a_{i j}, a_{i j}\right)\right] \\
& =-1+\frac{1}{n} \prod_{j=1}^{d}\left(1+\xi_{j} R_{\max }\right),
\end{aligned}
$$

where the inequality is obtained by omitting the $i \neq i^{\prime}$ terms which are nonnegative in the double sum in (35). This lower bound on $\left[e^{\mathrm{w}}\left(P_{n}, \mathcal{H}_{d, \eta, \boldsymbol{\xi}}^{\mathrm{wav}}\right)\right]^{2}$ is also a lower bound on $\left[e^{\mathrm{w}}\left(P_{n}, \mathcal{H}_{d, \eta, \boldsymbol{\beta}}^{\text {wav }}\right)\right]^{2}$ due to (36). Thus we have the following lemma.

Lemma 5. For any $n$ point set $P_{n} \subset[0,1)^{d}$, we have

$$
\left[e^{\mathrm{w}}\left(P_{n}, \mathcal{H}_{d, \eta, \boldsymbol{\beta}}^{\mathrm{wav}}\right)\right]^{2} \geq-1+\frac{1}{n} \prod_{j=1}^{d}\left(1+\mu \beta_{j} R_{\max }\right),
$$

where $R_{\max }$ is given in (37) and $\mu$ is defined by (38).

2.5. QMC strong tractability. Based on the lemmas of the previous subsections, the following theorem gives necessary and sufficient conditions for QMC strong tractability in the randomized worst-case setting. The necessary conditions hold for any set and the sufficient conditions hold for Niederreiter nets and sequences.

Theorem 1. Let $\mathcal{H}_{d, \eta, \boldsymbol{\beta}}^{\mathrm{wav}}$ be defined in (9) with $\omega_{u \kappa}$ defined in (8). Let $P_{n}^{N-\text { net }}$ denote a Niederreiter $(t, m, d)$-net in base $b$, and let $P_{n}^{N-\operatorname{seq}}$ denote the set of the first $n$ points of a Niederreiter $(t, d)$-sequence in base $b$.

(i) If multivariate integration in $\mathcal{H}_{d, \eta, \boldsymbol{\beta}}^{\text {wav }}$ in the randomized worst-case setting is QMC strongly tractable, then the weights $\beta_{j}$ in (8) must satisfy

$$
\sum_{j=1}^{\infty} \beta_{j}<\infty \text {. }
$$

(ii) If the $\beta_{j}$ in (8) satisfy

$$
\sum_{j=1}^{\infty} \beta_{j}\left(j \log _{b} j\right)^{1+\eta}<\infty
$$

then for any $\delta>0$, there exists a constant $C_{\delta}$ such that

$$
e^{\mathrm{w}}\left(P_{n}^{N-\mathrm{net}}, \mathcal{H}_{d, \eta, \boldsymbol{\beta}}^{\mathrm{wav}}\right) \leq C_{\delta} n^{-(1+\eta) / 2+\delta} .
$$

Consequently, multivariate integration in $\mathcal{H}_{d, \eta, \boldsymbol{\beta}}^{\text {wav }}$ in the randomized worstcase setting is $Q M C$ strongly tractable, and the $\varepsilon$-exponent of strong tractability is at most $2 /(1+\eta)$ for the scrambled Niederreiter $(t, m, d)$-nets.

(iii) If the $\beta_{j}$ in (8) satisfy (40), then for any $\delta>0$, there exists a constant $C_{\delta}$ such that

$$
e^{\mathrm{w}}\left(P_{n}^{N-\mathrm{seq}}, \mathcal{H}_{d, \eta, \boldsymbol{\beta}}^{\mathrm{wav}}\right) \leq C_{\delta} n^{-(1+\bar{\eta}) / 2+\delta},
$$

where $\bar{\eta}=\min (\eta, 1)$. Consequently, multivariate integration in $\mathcal{H}_{d, \eta, \boldsymbol{\beta}}^{\text {wav }}$ in the randomized worst-case setting is QMC strongly tractable, and the $\varepsilon$ exponent of strong tractability is at most $2 /(1+\bar{\eta})$ for the first $n$ points of a scrambled Niederreiter $(t, d)$-sequence. 
Proof. First the necessary condition is proved. For any $\varepsilon \in(0,1)$ and $d \geq 1$, let $n^{\mathrm{w}}(\varepsilon, d)$ denote the smallest number of points $n$ required in a QMC rule to ensure that the minimal $e^{\mathrm{w}}\left(P_{n}, \mathcal{H}_{d, \eta, \boldsymbol{\beta}}^{\mathrm{wav}}\right)$ is no larger than $\varepsilon e^{\mathrm{w}}\left(\emptyset, \mathcal{H}_{d, \eta, \boldsymbol{\beta}}^{\mathrm{wav}}\right)$. Since the initial error $e^{\mathrm{w}}\left(\emptyset, \mathcal{H}_{d, \eta, \boldsymbol{\beta}}^{\text {wav }}\right)$ satisfies

$$
\left[e^{\mathrm{w}}\left(\emptyset, \mathcal{H}_{d, \eta, \boldsymbol{\beta}}^{\mathrm{wav}}\right)\right]^{2}=\int_{[0,1)^{2 d}} K_{d, \eta, \boldsymbol{\beta}}^{\mathrm{sc}}(\mathbf{x}, \mathbf{y}) d \mathbf{x} d \mathbf{y}=\omega_{\emptyset}=1
$$

it follows from Lemma 5 that for QMC rules

$$
n^{\mathrm{w}}(\varepsilon, d) \geq \frac{1}{1+\varepsilon^{2}} \prod_{j=1}^{d}\left(1+\mu \beta_{j} R_{\max }\right)=\frac{1}{1+\varepsilon^{2}} \exp \left(\sum_{j=1}^{d} \log \left(1+\mu \beta_{j} R_{\max }\right)\right) .
$$

Let $M=\mu R_{\max } \sup _{j} \beta_{j}$. Using the fact that

$$
\log (1+x) \geq c x \quad \text { for } x \in[0, M], \quad \text { where } \quad c=\frac{\log (1+M)}{M},
$$

it follows that

$$
n^{\mathrm{w}}(\varepsilon, d) \geq \frac{1}{1+\varepsilon^{2}} \exp \left(c \mu R_{\max } \sum_{j=1}^{d} \beta_{j}\right) .
$$

Thus, $n^{\mathrm{w}}(\varepsilon, d)$ is bounded only if $\sum_{j=1}^{\infty} \beta_{j}<\infty$.

Next, we prove the sufficient conditions. If the $\beta_{j}$ satisfy condition (40), then for any fixed $\delta>0$ there exists an integer $\ell>0$ such that

$$
\widetilde{\theta} \sum_{j=\ell+1}^{\infty} \beta_{j}\left[j \log _{b}(j+b)\right]^{1+\eta}<\delta
$$

where $\widetilde{\theta}$ is defined as in Lemma 4, Define

$$
\begin{gathered}
\widetilde{C}_{\delta}=\min \left\{1,\left(\widetilde{\theta} \sum_{j=1}^{\ell} \beta_{j}\left[j \log _{b}(j+b)\right]^{1+\eta}\right)^{-1} \delta\right\}, \\
\alpha_{j}= \begin{cases}\widetilde{C}_{\delta} \widetilde{\theta} \beta_{j}, & j=1, \ldots, \ell, \\
\widetilde{\theta} \beta_{j}, & j=\ell+1, \ell+2, \ldots\end{cases}
\end{gathered}
$$

Note that $\alpha_{u}=\prod_{j \in u} \alpha_{j} \geq \widetilde{C}_{\delta}^{\ell} \widetilde{\theta}^{|u|} \beta_{u}$ and

$$
\begin{aligned}
\sum_{j=1}^{\infty} \alpha_{j}\left[j \log _{b}(j+b)\right]^{1+\eta}= & \widetilde{C}_{\delta} \widetilde{\theta} \sum_{j=1}^{\ell} \beta_{j}\left[j \log _{b}(j+b)\right]^{1+\eta} \\
& +\widetilde{\theta} \sum_{j=\ell+1}^{\infty} \beta_{j}\left[j \log _{b}(j+b)\right]^{1+\eta} \leq 2 \delta .
\end{aligned}
$$


Therefore, for a Niederreiter $(t, m, d)$-net, from (32) we have

$$
\begin{aligned}
& {\left[e^{\mathrm{w}}\left(P_{n}^{\mathrm{N}-\mathrm{net}}, \mathcal{H}_{d, \eta, \boldsymbol{\beta}}^{\mathrm{wav}}\right)\right]^{2}} \\
& \quad \leq C_{1} \widetilde{C}_{\delta}^{-\ell} n^{-(1+\eta)} \sum_{u \neq \emptyset} \alpha_{u} \prod_{j \in u}\left\{\left[j \log _{b}(j+b)\right]^{1+\eta} \log n\right\} \\
& \quad \leq C_{1} \widetilde{C}_{\delta}^{-\ell} n^{-(1+\eta)} \prod_{j=1}^{d}\left\{1+\alpha_{j}\left[j \log _{b}(j+b)\right]^{1+\eta} \log n\right\} \\
& \quad=C_{1} \widetilde{C}_{\delta}^{-\ell} n^{-(1+\eta)} \exp \left\{\sum_{j=1}^{d} \log \left(1+\alpha_{j}\left[j \log _{b}(j+b)\right]^{1+\eta} \log n\right)\right\} \\
& \quad \leq C_{1} \widetilde{C}_{\delta}^{-\ell} n^{-(1+\eta)} \exp \left\{\sum_{j=1}^{d} \alpha_{j}\left[j \log _{b}(j+b)\right]^{1+\eta} \log n\right\} \\
& \quad \leq C_{1} \widetilde{C}_{\delta}^{-\ell} n^{-(1+\eta)} \exp \{2 \delta \log n\}=C_{1 \delta}^{2} n^{-(1+\eta)+2 \delta},
\end{aligned}
$$

where $C_{1 \delta}=\sqrt{C_{1} \widetilde{C}_{\delta}^{-\ell}}$. For any $\varepsilon \in(0,1)$, let $n^{\mathrm{w}}(\varepsilon, d)$ denote the smallest number of points $n$ required in a QMC rule to ensure that the minimal $e^{\mathrm{w}}\left(P_{n}^{\mathrm{N}-\mathrm{net}}, \mathcal{H}_{d, \eta, \boldsymbol{\beta}}^{\mathrm{wav}}\right)$ is no larger than $\varepsilon e^{\mathrm{w}}\left(\emptyset, \mathcal{H}_{d, \eta, \boldsymbol{\beta}}^{\mathrm{wav}}\right)$. Since $e^{\mathrm{w}}\left(\emptyset, \mathcal{H}_{d, \eta, \boldsymbol{\beta}}^{\mathrm{wav}}\right)=1$, it follows from the last inequality with arbitrary $\delta>0$ that

$$
n^{\mathrm{w}}(\varepsilon, d) \leq C_{1 \delta} \varepsilon^{-2 /(1+\eta)} \text { for all } \quad d \geq 1 .
$$

This completes the proof of (ii) under condition (40).

For the first $n$ points of a Niederreiter $(t, d)$-sequence, from (33) we have

$$
\left[e^{\mathrm{w}}\left(P_{n}^{\mathrm{N}-\mathrm{seq}}, \mathcal{H}_{d, \eta, \boldsymbol{\beta}}^{\mathrm{wav}}\right)\right]^{2} \leq C_{2} \widetilde{C}_{\delta}^{-\ell} n^{-(1+\bar{\eta})} \sum_{u \neq \emptyset} \alpha_{u} \prod_{j \in u}\left\{\left[j \log _{b}(j+b)\right]^{1+\eta} \log n\right\} .
$$

By a similar argument for $e^{\mathrm{w}}\left(P_{n}^{\mathrm{N}-\mathrm{net}}, \mathcal{H}_{d, \eta, \boldsymbol{\beta}}^{\mathrm{wav}}\right)$ above, we obtain

$$
\left[e^{\mathrm{w}}\left(P_{n}^{\mathrm{N}-\mathrm{seq}}, \mathcal{H}_{d, \eta, \boldsymbol{\beta}}^{\mathrm{wav}}\right)\right]^{2} \leq C_{2 \delta}^{2} n^{-(1+\bar{\eta})+2 \delta},
$$

where $C_{2 \delta}=\sqrt{C_{2} \widetilde{C}_{\delta}^{-\ell}}$. This leads to the results in (iii).

Remark 1. The results for Niederreiter $(t, m, d)$-nets also hold for Niederreiter $(\lambda, t, m, d)$-nets with $\lambda \in\{0,1, \ldots, b-1\}$ and $n=\lambda b^{m}$. The definition of a $(\lambda, t, m, d)$-net is given in [14.

Remark 2. For the Haar-like wavelet case, the digital smoothness parameter $\eta$ plays an important role in the error analysis. For $0<\eta \leq 1$, we can achieve, roughly, the convergence rate of $\mathcal{O}\left(n^{-(1+\eta) / 2}\right)$ regardless of whether $n=\lambda b^{m}$ or not. However, for $\eta>1$, the rates of convergence are quite different for a $(\lambda, t, m, d)$-net and for the first $n$ points of a $(t, d)$-sequence with $n \neq \lambda b^{m}$. In fact, in the latter case additional smoothness does not improve the convergence rate at all. For QMC rules that use scrambled sequences adding some additional points into a $(\lambda, t, m, d)$-net may sometimes cause a large loss of efficiency no matter how smooth the integrand is [26. The reason for the difference in performance in nets and sequences for $\eta>1$ is that nets integrate coarse Haar wavelets exactly, whereas sequences do not. For nets the quadrature error comes from the inability to integrate fine Haar wavelets. Sequences also do poorly in integrating fine Haar wavelets, but they have at least an $\mathcal{O}\left(n^{-1}\right)$ error in integrating the coarse Haar wavelets as well. 
Remark 3. As has been noted elsewhere, digital smoothness is not the same as smoothness in the usual sense. However, the space of integrands $\mathcal{H}_{d, \gamma}^{\mathrm{SH}}$ in the next section with square integrable first derivatives corresponds roughly to $\mathcal{H}_{d, \eta, \boldsymbol{\beta}}^{\text {wav }}$ with digital smoothness parameter $\eta=1$.

\section{INTEGRATION OVER $\mathcal{H}_{d, \boldsymbol{\gamma}}^{\mathrm{SH}}$ IN THE RANDOMIZED WORST-CASE SETTING}

In this section we consider the QMC strong tractability problem of multivariate integration using scrambled Niederreiter points in the randomized worst-case setting over the weighted Sobolev-Hilbert space $\mathcal{H}_{d, \gamma}^{\mathrm{SH}}$.

The weighted Sobolev-Hilbert space is a tensor product of $d$ different SobolevHilbert spaces $\mathcal{H}_{1, \gamma}^{\mathrm{SH}}$ defined for absolutely continuous univariate functions on $[0,1)$ with squared integrable derivative. The inner products in $\mathcal{H}_{1, \gamma}^{\mathrm{SH}}$ have the form

$$
\langle f, g\rangle_{\mathrm{SH}, 1, \gamma}=\int_{0}^{1} f(x) d x \int_{0}^{1} g(x) d x+\gamma^{-1} \int_{0}^{1} f^{\prime}(x) g^{\prime}(x) d x,
$$

where $\gamma>0$ is a parameter or weight which may take different values in the different components of the tenor product. Following Sloan and Woźniakowski [17], we suppose functions depend on the coordinates $x_{1}, x_{2}, \ldots, x_{d}$ in such a way that $x_{1}$ is the most important, $x_{2}$ the next, and so on; and we quantify this by associating weights $\gamma=\left(\gamma_{1}, \ldots, \gamma_{d}\right)$, with

$$
\gamma_{1} \geq \gamma_{2} \geq \cdots \geq \gamma_{d}>0
$$

to the successive coordinate directions. Our tensor product space is then

$$
\mathcal{H}_{d, \gamma}^{\mathrm{SH}}=\mathcal{H}_{1, \gamma_{1}}^{\mathrm{SH}} \otimes \mathcal{H}_{1, \gamma_{2}}^{\mathrm{SH}} \otimes \cdots \otimes \mathcal{H}_{1, \gamma_{d}}^{\mathrm{SH}},
$$

and the inner product in $\mathcal{H}_{d, \boldsymbol{\gamma}}^{\mathrm{SH}}$ is

$$
\begin{aligned}
\langle f, g\rangle_{\mathrm{SH}, d, \boldsymbol{\gamma}}=\sum_{u \subseteq \mathcal{A}} \gamma_{u}^{-1} \int_{[0,1)|u|} & \left(\int_{[0,1)^{d-|u|}} \frac{\partial^{|u|} f(\mathbf{x})}{\partial \mathbf{x}_{u}} d \mathbf{x}_{\mathcal{A}-u}\right) \\
& \times\left(\int_{[0,1)^{d-|u|}} \frac{\partial^{|u|} g(\mathbf{x})}{\partial \mathbf{x}_{u}} d \mathbf{x}_{\mathcal{A}-u}\right) d \mathbf{x}_{u}
\end{aligned}
$$

where $\gamma_{\emptyset}=1$ and $\gamma_{u}=\prod_{j \in u} \gamma_{j}$ for any $u \neq \emptyset$. The squared norm in $\mathcal{H}_{d, \gamma}^{\mathrm{SH}}$ is

$$
\|f\|_{\mathrm{SH}, d, \boldsymbol{\gamma}}^{2}=\sum_{u \subseteq \mathcal{A}} \gamma_{u}^{-1} \int_{[0,1)|u|}\left(\int_{[0,1)} d-|u| \frac{\partial^{|u|} f(\mathbf{x})}{\partial \mathbf{x}_{u}} \mathbf{x}_{\mathcal{A}-u}\right)^{2} d \mathbf{x}_{u} .
$$

It can be verified that the reproducing kernel for this space is

$$
K_{d, \gamma}^{\mathrm{SH}}(\mathbf{x}, \mathbf{y})=\prod_{j=1}^{d}\left[1+\gamma_{j}\left(\frac{1}{3}+\frac{x_{j}^{2}+y_{j}^{2}}{2}-\max \left(x_{j}, y_{j}\right)\right)\right] .
$$

This space is considered by Sloan and Woźniakowski [19, Section 3.3] for Monte Carlo quadrature. For the special case where $\gamma_{j}=1$ for $j=1, \ldots, d$, the space $\mathcal{H}_{d, \gamma}^{\mathrm{SH}}$ becomes the Sobolev-Hilbert space described by Wahba [21, Chapter 10]. 
We now consider integration for the weighted Sobolev-Hilbert space $\mathcal{H}_{d, \gamma}^{\mathrm{SH}}$ with reproducing kernel $K_{d, \gamma}^{\mathrm{SH}}$. The randomized worst-case error defined in (11) for $\mathcal{H}_{d, \gamma}^{\mathrm{SH}}$ is

$$
e^{\mathrm{w}}\left(P_{n}, \mathcal{H}_{d, \boldsymbol{\gamma}}^{\mathrm{SH}}\right)=\left(E\left[\sup _{\|f\|_{\mathrm{SH}, d, \boldsymbol{\gamma}} \leq 1} \mid I_{d}(f)-Q_{n, d}\left(f, P_{n}^{\mathrm{sc}}\right)\right]^{2}\right)^{1 / 2} .
$$

From Hickernell and Yue 8 we have

$$
e^{\mathrm{w}}\left(P_{n}, \mathcal{H}_{d, \gamma}^{\mathrm{SH}}\right)=e^{\mathrm{w}}\left(P_{n}, \mathcal{H}_{d, \eta, \boldsymbol{\beta}}^{\mathrm{wav}}\right)
$$

with $\eta=1$ and $\beta_{j}=\gamma_{j}(6 b)^{-1}$ for $j=1, \cdots, d$. The initial error $e^{\mathrm{w}}\left(\emptyset, \mathcal{H}_{d, \gamma}^{\mathrm{SH}}\right)$ satisfies

$$
\left[e^{\mathrm{w}}\left(\emptyset, \mathcal{H}_{d, \boldsymbol{\gamma}}^{\mathrm{SH}}\right)\right]^{2}=\int_{[0,1)^{2 d}} K_{d, \boldsymbol{\gamma}}^{\mathrm{SH}}(\mathbf{x}, \mathbf{y}) d \mathbf{x} d \mathbf{y}=\omega_{\emptyset}=1
$$

Applying the results in Theorem 1 replacing $\beta_{j}$ and $\eta$ by $\gamma_{j}(6 b)^{-1}$ and 1 , respectively, yields the following sufficient conditions for strong tractability for integration in $\mathcal{H}_{d, \gamma}^{\mathrm{SH}}$ using the scrambled Niederreiter points. Necessary conditions for QMC strong tractability have been proven by Sloan and Woźniakowski [17, 18] and Woźniakowski [24].

Theorem 2. Let $\mathcal{H}_{d, \gamma}^{\mathrm{SH}}$ be the weighted Sobolev-Hilbert space defined in (41) with reproducing kernel $K_{d, \gamma}^{\mathrm{SH}}$ given in (44). If multivariate integration in the randomized worst-case setting is QMC strongly tractable, then the weights must satisfy

$$
\sum_{j=1}^{\infty} \gamma_{j}<\infty
$$

Now, assume that the scrambled QMC quadrature employs the first $n$ points of the Niederreiter $(t, d)$-sequence in prime power base $b$, whether $n=b^{m}$ or not. If the weights $\gamma_{j}$ satisfy

$$
\sum_{j=1}^{\infty} \gamma_{j}\left(j \log _{b} j\right)^{2}<\infty
$$

then for any $\delta>0$, there exists a constant $C_{\delta}$ such that

$$
e^{\mathrm{w}}\left(P_{n}^{N-\mathrm{seq}}, \mathcal{H}_{d, \gamma}^{\mathrm{SH}}\right) \leq C_{\delta} n^{-1+\delta} .
$$

Consequently, multivariate integration in $\mathcal{H}_{d, \gamma}^{\mathrm{SH}}$ in the randomized worst-case setting is QMC strongly tractable, and the E-exponent of strong tractability is at most 1 for the scrambled Niederreiter sequence, regardless of whether $n=b^{m}$ or not.

Remark 4. For the weighted Sobolev spaces, $\mathcal{H}_{d, \gamma}^{\text {Sob }}$, considered by, e.g., Sloan and Woźniakowski [17] and Wang [23], the reproducing kernel is given by

$$
K_{d, \gamma}^{\mathrm{Sob}}(\mathbf{x}, \mathbf{y})=\prod_{j=1}^{d}\left[1+\gamma_{j}\left(1-\max \left(x_{j}, y_{j}\right)\right)\right] .
$$

We find that the associated scramble-invariant kernel, $K_{d, \gamma}^{\text {Sob,sc }}$, is of the form (11) with $\omega_{u \kappa}$ given by

$$
\omega_{u \kappa}=\left[\prod_{j=1}^{d}\left(1+\frac{\gamma_{j}}{3}\right)\right] \cdot\left[\prod_{j \in u} \frac{\gamma_{j}}{6 b\left(1+\gamma_{j} / 3\right)}\right] b^{-2\|\boldsymbol{\kappa}\|_{1}} .
$$


The initial error for this space satisfies

$$
\left[e^{\mathrm{w}}\left(\emptyset, \mathcal{H}_{d, \gamma}^{\mathrm{Sob}}\right)\right]^{2}=\prod_{j=1}^{d}\left(1+\frac{\gamma_{j}}{3}\right) .
$$

Therefore, if the weights $\gamma_{j}$ satisfy

$$
\prod_{j=1}^{\infty}\left(1+\frac{\gamma_{j}}{3}\right)<\infty \text { and } \sum_{j=1}^{\infty} \frac{\gamma_{j}}{\left(1+\gamma_{j} / 3\right)}\left(j \log _{b} j\right)^{2}<\infty,
$$

then for any $\delta>0$, there exists a constant $C_{\delta}$ such that

$$
e^{\mathrm{w}}\left(P_{n}^{\mathrm{N}-\mathrm{seq}}, \mathcal{H}_{d, \gamma}^{\mathrm{Sob}}\right) \leq C_{\delta} n^{-1+\delta} .
$$

Note that the condition in 46 is equivalent to the condition

$$
\sum_{j=1}^{\infty} \gamma_{j}\left(j \log _{b} j\right)^{2}<\infty
$$

This condition is weaker compared to the following condition 23 of strong tractability of integration using the deterministic Niederreiter sequence in this space:

$$
\sum_{j=1}^{\infty} \gamma_{j}^{1 / 2} j \log _{b} j<\infty
$$

but with the same $\varepsilon$-exponent.

\section{Integration OVer $\mathcal{H}_{d, \eta, \boldsymbol{\beta}}^{\text {wav }}$ IN THE RANDOMized SETting}

This section considers the QMC strong tractability problems in the randomized setting for the weighted Hilbert space $\mathcal{H}_{d, \eta, \boldsymbol{\beta}}^{\text {wav }}$ with reproducing kernel $K_{d, \eta, \boldsymbol{\beta}}^{\mathrm{sc}}$. The randomized error defined in (2) for $\mathcal{H}_{d, \eta, \boldsymbol{\beta}}^{\mathrm{wav}}$ is

$$
e^{\mathrm{r}}\left(P_{n}, \mathcal{H}_{d, \eta, \boldsymbol{\beta}}^{\mathrm{wav}}\right)=\left(\sup _{\|f\|_{\mathrm{wav}, d, \eta, \boldsymbol{\beta}} \leq 1} E\left|I_{d}(f)-Q_{n, d}\left(f, P_{n}^{\mathrm{sc}}\right)\right|^{2}\right)^{1 / 2},
$$

and then the initial error is

$$
e^{\mathrm{r}}\left(\emptyset, \mathcal{H}_{d, \eta, \boldsymbol{\beta}}^{\mathrm{wav}}\right)=\sup _{\|f\|_{\text {wav }, d, \eta, \boldsymbol{\beta}} \leq 1}\left|I_{d}(f)\right| .
$$

It is known from Heinrich, Hickernell and Yue [1] that the randomized error $e^{\mathrm{r}}\left(P_{n}, \mathcal{H}_{d, \eta, \boldsymbol{\beta}}^{\text {wav }}\right)$ can be expressed as

$$
e^{\mathrm{r}}\left(P_{n}, \mathcal{H}_{d, \eta, \boldsymbol{\beta}}^{\mathrm{wav}}\right)=\left(\max _{u, \boldsymbol{\kappa}}\left\{n^{-1} \omega_{u \kappa} \Gamma_{u \kappa}\left(P_{n}\right)\right\}\right)^{1 / 2},
$$

where $\omega_{u \kappa}$ and $\Gamma_{u \kappa}$ are as defined in (8) and (15), respectively. If $P_{n}$ consists of the Niederreiter points, we have the following results.

Theorem 3. Suppose that the space $\mathcal{H}_{d, \eta, \boldsymbol{\beta}}^{\text {wav }}$ is defined as in (9) where the $\omega_{u \kappa}$ are given by (8). Let $P_{n}^{N-\text { net }}$ and $P_{n}^{N-\text { seq }}$ be defined as in Theorem 1 .

(i) If the $\beta_{j}$ in (8) satisfy

$$
\sum_{j=1}^{\infty}\left(\log \left[\mu_{1} \beta_{j}\left(j \log _{b} j\right)^{2+\eta}\right]\right)_{+}<\infty,
$$


where $\mu_{1}=b^{3+2 \eta}(b+1) /(b-1)$ and $(x)_{+}=x$ if $x \geq 0$ and 0 otherwise, then there exists a constant $C$ such that

$$
e^{\mathrm{r}}\left(P_{n}^{N-\mathrm{net}}, \mathcal{H}_{d, \eta, \boldsymbol{\beta}}^{\mathrm{wav}}\right) \leq C n^{-(2+\eta) / 2}, \quad \forall \eta>0 .
$$

Consequently, multivariate integration in $\mathcal{H}_{d, \eta, \boldsymbol{\beta}}^{\mathrm{wav}}$ in the randomized setting is $Q M C$ strongly tractable, and the $\varepsilon$-exponent of strong tractability is $2 /(2+\eta)$ for the scrambled Niederreiter nets.

(ii) If the $\beta_{j}$ in (8) satisfy

$$
\sum_{j=1}^{\infty}\left(\log \left[\mu_{2} \beta_{j}\left(j \log _{b} j\right)^{2}\right]\right)_{+}<\infty
$$

where $\mu_{2}=b^{3}(b+1) /(b-1)$, then there exists a constant $C$ such that

$$
e^{\mathrm{r}}\left(P_{n}^{N-\operatorname{seq}}, \mathcal{H}_{d, \eta, \boldsymbol{\beta}}^{\mathrm{wav}}\right) \leq C n^{-1}, \quad \forall \eta>0 .
$$

Consequently, multivariate integration in $\mathcal{H}_{d, \eta, \boldsymbol{\beta}}^{\text {wav }}$ in the randomized setting is QMC strongly tractable, and the $\varepsilon$-exponent of strong tractability is 1 for the first $n$ points of a scrambled Niederreiter sequence.

Proof. For the Niederreiter $(t, m, d)$-net $P_{n}^{\mathrm{N}-\text { net}}$, making use of (8), (17) and (31), we have

$$
\begin{aligned}
\max _{u, \kappa} & \left\{n^{-1} \omega_{u \kappa} \Gamma_{u \kappa}\left(P_{n}^{\mathrm{N}-\mathrm{net}}\right)\right\} \\
\leq & n^{-1} \max _{\substack{u, k ! \\
k \geq m-t_{u}-|u|+1}}\left\{\beta_{u} b^{-(1+\eta) k+t_{u}}\left(\frac{b+1}{b-1}\right)^{|u|}\right\} \\
& \leq n^{-(2+\eta)} b^{-1-\eta} \max _{u}\left\{\beta_{u} b^{(2+\eta) t_{u}}\left[\frac{(b+1) b^{1+\eta}}{b-1}\right]^{|u|}\right\} \\
& \leq n^{-(2+\eta)} b^{-1-\eta} \max _{u}\left\{\prod_{j \in u}\left[\mu_{1} \beta_{j}\left(j \log _{b}(j+b)\right)^{2+\eta}\right]\right\},
\end{aligned}
$$

where $\mu_{1}=b^{3+2 \eta}(b+1) /(b-1)$. If condition (49) holds, then

$$
\prod_{j=1}^{\infty}\left[\mu_{1} \beta_{j}\left(j \log _{b}(j+b)\right)^{2+\eta}\right]<\infty
$$

and there exists some constant $C_{*}$ such that

$$
\prod_{j \in u}\left[\mu_{1} \beta_{j}\left(j \log _{b}(j+b)\right)^{2+\eta}\right] \leq C_{*}, \quad \forall u .
$$

Therefore, from (48) we have

$$
e^{\mathrm{r}}\left(P_{n}^{\mathrm{N}-\mathrm{net}}, \mathcal{H}_{d, \eta, \boldsymbol{\beta}}^{\mathrm{wav}}\right) \leq C n^{-(2+\eta) / 2},
$$

where $C=\sqrt{C_{*} b^{-1-\eta}}$. This completes the proof under condition (49). 
For the first $n$ points of a Niederreiter $(t, d)$-sequence, $P_{n}^{\mathrm{N}-\text { seq }}$, let $q$ be the largest integer such that $b^{q} \leq n$. Making use of (8), (18) and (31), we have for any $\eta>0$

$$
\begin{aligned}
\max _{u, \kappa} & \left\{n^{-1} \omega_{u \kappa} \Gamma_{u \kappa}\left(P_{n}^{\mathrm{N}-\mathrm{seq}}\right)\right\} \\
& =\max _{u, \boldsymbol{\kappa}}\left\{n^{-1} \beta_{u} b^{-(1+\eta)\|\boldsymbol{\kappa}\|_{1}} \Gamma_{u \kappa}\left(P_{n}^{\mathrm{N}-\mathrm{seq}}\right)\right\} \\
& \leq \max _{u, \boldsymbol{\kappa}}\left\{n^{-1} \beta_{u} b^{-\|\boldsymbol{\kappa}\|_{1}} \Gamma_{u \kappa}\left(P_{n}^{\mathrm{N}-\mathrm{seq}}\right)\right\} \\
& \leq n^{-2} \frac{b+1}{b-1} \max _{u, \kappa}\left\{\beta_{u}\left(\frac{b+1}{b-1}\right)^{|u|} b^{t_{u}} b^{\min \left(t_{u}+|u|+\|\boldsymbol{\kappa}\|_{1}, q+1\right)-\|\boldsymbol{\kappa}\|_{1}}\right\} \\
& =n^{-2} \frac{b+1}{b-1} \max _{u}\left\{\beta_{u}\left(\frac{b+1}{b-1}\right)^{|u|} b^{t_{u}} b^{t_{u}+|u|}\right\} \\
& \leq n^{-2} \frac{b+1}{b-1} \max _{u}\left\{\prod_{j \in u}\left[\mu_{2} \beta_{j}\left(j \log _{b}(j+b)\right)^{2}\right]\right\},
\end{aligned}
$$

where $\mu_{2}=b^{3}(b+1) /(b-1)$. If condition (50) holds, then

$$
\prod_{j=1}^{\infty}\left[\mu_{2} \beta_{j}\left(j \log _{b}(j+b)\right)^{2}\right]<\infty
$$

and there exists some constant $C_{*}$ such that

$$
\prod_{j \in u}\left[\mu_{2} \beta_{j}\left(j \log _{b}(j+b)\right)^{2}\right] \leq C_{*}, \quad \forall u .
$$

It follows from (48) that

$$
e^{\mathrm{r}}\left(P_{n}^{\mathrm{N}-\mathrm{seq}}, \mathcal{H}_{d, \eta, \boldsymbol{\beta}}^{\mathrm{wav}}\right) \leq C n^{-1}, \quad \forall \eta>0,
$$

where $C=\sqrt{C_{*}(b+1) /(b-1)}$. This completes the proof under condition (50).

Remark 5. Note that for the Niederreiter sequences, for example, the condition in (51) holds if there exists an integer $j_{0}$ such that $0<\beta_{j} \leq\left(1+j^{-\alpha}\right) /\left[\mu_{2}\left(j \log _{b} j\right)^{2}\right]$ for all $j \geq j_{0}$, where $\alpha>1$ is a constant.

\section{Integration Over $\mathcal{H}_{d, \gamma}^{\mathrm{SH}}$ IN THE RANDOMized SETting}

In this section we consider the QMC strong tractability problem in the randomized setting over the Sobolev-Hilbert space $\mathcal{H}_{d, \gamma}^{\mathrm{SH}}$ with the norm defined in (43). The following lemma gives an upper bound on the randomized error for any scrambled QMC quadrature.

Lemma 6. Let $\mathcal{H}_{d, \gamma}^{\mathrm{SH}}$ be the weighted Sobolev-Hilbert space with the inner product and norm defined by (42) and (43). If the quadrature employs a scrambled version of the set $P_{n}$ of $n$ points in $[0,1)^{d}$, then

$$
\left[e^{\mathrm{r}}\left(P_{n}, \mathcal{H}_{d, \gamma}^{\mathrm{SH}}\right)\right]^{2} \leq \max _{u \neq \emptyset}\left\{n^{-1} \gamma_{u}\left(\frac{b-1}{3}\right)^{|u|} \sum_{\kappa} b^{-2\|\boldsymbol{\kappa}\|_{1}} \Gamma_{u \kappa}\left(P_{n}\right)\right\} .
$$


Proof. For any function $f$ in $\mathcal{H}_{d, \gamma}^{\mathrm{SH}}$, it can be expanded in a series in terms of the Haar-like wavelets defined in (6), i.e.,

(53) $f(\mathbf{x})=\sum_{u \kappa \tau \mathbf{c}} \hat{f}_{u \kappa \tau \mathbf{c}} \psi_{u \kappa \tau \mathbf{c}}(\mathbf{x}), \quad$ where $\quad \hat{f}_{u \kappa \tau \mathbf{c}}=\int_{[0,1)^{d}} f(\mathbf{x}) \psi_{u \kappa \tau \mathbf{c}}(\mathbf{x}) d \mathbf{x}$.

Let $P_{n}^{\text {sc }}$ be a scrambled version of $P_{n}$. Note from Owen (1997a) that

$$
E\left|I_{d}(f)-Q_{n, d}\left(f, P_{n}^{\mathrm{sc}}\right)\right|^{2}=\operatorname{var}\left(Q_{n, d}\right)=n^{-1} \sum_{u \neq \emptyset} \sum_{\kappa} \sigma_{u \kappa}^{2}(f) \Gamma_{u \kappa}\left(P_{n}\right),
$$

where $\sigma_{u \kappa}^{2}$ is given by

$$
\sigma_{u \kappa}^{2}(f)=\sum_{\boldsymbol{\tau}, \mathbf{c}, \mathbf{c}^{\prime}} \hat{f}_{u \kappa \tau \mathbf{c}} \hat{f}_{u \kappa \tau \mathbf{c}^{\prime}} \prod_{j \in u}\left(1_{c_{j}=c_{j^{\prime}}}-b^{-1}\right) .
$$

For any given $u, \boldsymbol{\kappa}, \boldsymbol{\tau}$ and $\mathbf{c}$ we define

$$
h_{u \kappa \tau \mathbf{c}}(\mathbf{x})=\prod_{j \in u} \int_{0}^{x_{j}} \psi_{\kappa_{j} \tau_{j} c_{j}}\left(t_{j}\right) d t_{j}, \quad \forall \mathbf{x} \in[0,1)^{d},
$$

where $\psi_{\kappa \tau c}$ is defined as in (5). In fact, $h_{u \kappa \tau \mathbf{c}}(\mathbf{x})$ depends only on $\mathbf{x}_{u} \in[0,1)^{|u|}$, i.e., $h_{u \kappa \tau \mathbf{c}}(\mathbf{x})=h_{u \kappa \tau \mathbf{c}}\left(\mathbf{x}_{u}\right)$. Define

$$
S_{u \kappa \tau}=\prod_{j \in u}\left[\frac{\tau_{j}}{b^{\kappa_{j}}}, \frac{\tau_{j}+1}{b^{\kappa_{j}}}\right) .
$$

Then $S_{u \kappa \tau}$ is the support point set of $h_{u \kappa \tau \mathbf{c}}\left(\mathbf{x}_{u}\right)$ in $[0,1)^{|u|}$, and

$$
\sum_{\tau} S_{u \kappa \tau}=[0,1)^{|u|}, \quad \forall u, \kappa .
$$

Making use of integration by parts, the coefficients $\hat{f}_{u \kappa \tau c}$ defined in (53) can be expressed as

$$
\begin{aligned}
\hat{f}_{u \kappa \tau \mathbf{c}} & =(-1)^{|u|} \int_{[0,1)^{d}} h_{u \kappa \tau \mathbf{c}}(\mathbf{x}) \frac{\partial^{|u|} f(\mathbf{x})}{\partial \mathbf{x}_{u}} d \mathbf{x} \\
& =(-1)^{|u|} \int_{S_{u \kappa \tau}} h_{u \kappa \tau \mathbf{c}}\left(\mathbf{x}_{u}\right) \int_{[0,1)^{d-|u|}} \frac{\partial^{|u|} f(\mathbf{x})}{\partial \mathbf{x}_{u}} d \mathbf{x}_{\mathcal{A}-u} d \mathbf{x}_{u} .
\end{aligned}
$$

Straightforward calculation from the definition of $h_{u \kappa \tau \mathbf{c}}$ gives

$$
\int_{S_{u \kappa \tau}} h_{u \kappa \tau \mathbf{c}}^{2}\left(\mathbf{x}_{u}\right) d \mathbf{x}_{u}=\prod_{j \in u} \frac{3 c_{j}^{2}-3(b-1) c_{j}+(b-1)^{2}}{3 b^{2 \kappa_{j}+3}} \leq(3 b)^{-|u|} b^{-2\|\boldsymbol{\kappa}\|_{1}} .
$$

Applying the Schwarz inequality in (57) and using the upper bound in (58) gives

$$
\left|\hat{f}_{u \kappa \tau \mathbf{c}}\right| \leq(3 b)^{-|u| / 2} b^{-\|\boldsymbol{\kappa}\|_{1}}\left[\int_{S_{u \kappa \tau}}\left(\int_{[0,1)^{d-|u|}} \frac{\partial^{|u|} f(\mathbf{x})}{\partial \mathbf{x}_{u}} d \mathbf{x}_{\mathcal{A}-u}\right)^{2} d \mathbf{x}_{u}\right]^{1 / 2} .
$$

Then from (55) and noting that $\prod_{j \in u}\left|1_{c_{j}=c_{j}^{\prime}}-b^{-1}\right| \leq\left(1-b^{-1}\right)^{|u|}$, we have

$$
\sigma_{u \kappa}^{2}(f) \leq\left(\frac{b-1}{3 b^{2}}\right)^{|u|} b^{-2\|\boldsymbol{\kappa}\|_{1}} \sum_{\tau, \mathbf{c}, \mathbf{c}^{\prime}} \int_{S_{u \kappa \boldsymbol{\tau}}}\left(\int_{[0,1)^{d-|u|}} \frac{\partial^{|u|} f(\mathbf{x})}{\partial \mathbf{x}_{u}} d \mathbf{x}_{\mathcal{A}-u}\right)^{2} d \mathbf{x}_{u} .
$$


Making use of the fact in (56) and $\sum_{\mathbf{c}, \mathbf{c}^{\prime}} 1=b^{2|u|}$ yield

$$
\sigma_{u \kappa}^{2}(f) \leq\left(\frac{b-1}{3}\right)^{|u|} b^{-2\|\boldsymbol{\kappa}\|_{1}} \int_{[0,1)^{|u|}}\left(\int_{[0,1)^{d-|u|}} \frac{\partial^{|u|} f(\mathbf{x})}{\partial \mathbf{x}_{u}} d \mathbf{x}_{\mathcal{A}-u}\right)^{2} d \mathbf{x}_{u} .
$$

This inequality together with (54) gives that

$$
\begin{aligned}
& E\left|I(f)-Q_{n, d}\left(f, P_{n}^{\mathrm{sc}}\right)\right|^{2} \\
& \quad \leq n^{-1} \sum_{u, \boldsymbol{\kappa}}\left(\frac{b-1}{3}\right)^{|u|} b^{-2\|\boldsymbol{\kappa}\|_{1}} \Gamma_{u \boldsymbol{\kappa}}\left(P_{n}\right) \int_{[0,1)^{|u|}}\left(\int_{[0,1)^{d-|u|}} \frac{\partial^{|u|} f(\mathbf{x})}{\partial \mathbf{x}_{u}} d \mathbf{x}_{\mathcal{A}-u}\right)^{2} d \mathbf{x}_{u} \\
& \quad \leq \max _{u}\left\{n^{-1} \gamma_{u}\left(\frac{b-1}{3}\right)^{|u|} \sum_{\boldsymbol{\kappa}} b^{-2\|\boldsymbol{\kappa}\|_{1}} \Gamma_{u \boldsymbol{\kappa}}\left(P_{n}\right)\right\}\|f\|_{\mathrm{SH}, d, \boldsymbol{\gamma}}^{2},
\end{aligned}
$$

where the norm in (43) is used. The result in (52) then follows.

From Lemma 6 and using the argument similar to that in the proof of Theorem 1. we can prove the following strong tractability results of multivariate integration in the weighted Sobolev-Hilbert space.

Theorem 4. Let $\mathcal{H}_{d, r}^{\mathrm{SH}}$ be the weighted Sobolev-Hilbert space defined in (41))-(44). Let $P_{n}^{N-\text { net }}$ and $P_{n}^{N-\text { seq }}$ be defined as in Theorem 1 .

(i) If the weights $\gamma_{j}$ satisfy

$$
\sum_{j=1}^{\infty} \gamma_{j}\left(j \log _{b} j\right)^{3}<\infty
$$

then for any $\delta>0$, there exists a constant $C_{\delta}$ such that

$$
e^{\mathrm{r}}\left(P_{n}^{N-\mathrm{net}}, \mathcal{H}_{d, \gamma}^{\mathrm{SH}}\right) \leq C_{\delta} n^{-3 / 2+\delta} .
$$

Consequently, multivariate integration in $\mathcal{H}_{d, \gamma}^{\mathrm{SH}}$ in the randomized setting is $Q M C$ strongly tractable, and the $\varepsilon$-exponent of strong tractability is at most $2 / 3$ for scrambled Niederreiter nets.

(ii) If the weights $\gamma_{j}$ satisfy

$$
\sum_{j=1}^{\infty} \gamma_{j}\left(j \log _{b} j\right)^{2}<\infty
$$

then for any $\delta>0$, there exists a constant $C_{\delta}$ such that

$$
e^{\mathrm{r}}\left(P_{n}^{N-\mathrm{seq}}, \mathcal{H}_{d, \gamma}^{\mathrm{SH}}\right) \leq C_{\delta} n^{-1+\delta} .
$$

Consequently, multivariate integration in $\mathcal{H}_{d, \gamma}^{\mathrm{SH}}$ in the randomized setting is $Q M C$ strongly tractable, and the $\varepsilon$-exponent of strong tractability is at most 1 for the first $n$ points of a scrambled Niederreiter sequence.

Remark 6. For this Sobolev-Hilbert space, Sloan and Woźniakowski [19, Section 3.3] show that Monte Carlo is strongly tractable in randomized settings if the weights $\gamma_{j}$ satisfy condition $\lim \sup _{d \rightarrow \infty} \sum_{j=1}^{d}\left(\ln \frac{\gamma_{j}}{\alpha}\right)_{+}<\infty$. However, the $\varepsilon$-exponent is 2 . Now, as shown in Theorem 4, if the $\gamma_{j}$ satisfy condition (59), then the $\varepsilon$-exponent is at most $2 / 3$ for quasi-Monte Carlo using scrambled Niederreiter nets, and if the $\gamma_{j}$ satisfy condition (60), then the $\varepsilon$-exponent is at most 1 for quasi-Monte Carlo using scrambled Niederreiter sequences. 
Remark 7. To the authors' knowledge this is the first time that a convergence rate of $n^{-3 / 2}$ in the randomized setting has been shown for $(t, m, d)$-nets with so little smoothness required. In [15] it is assumed that the mixed partial derivatives of the integrand of up to order one in each coordinate satisfy a Lipschitz condition. Here in (43) it need only be assumed that these derivatives are square integrable.

Remark 8. As mentioned in Remark 3 , the space $\mathcal{H}_{d, \eta, \boldsymbol{\beta}}^{\text {wav }}$ with $\eta=1$ is similar to $\mathcal{H}_{d, \gamma}^{\mathrm{SH}}$. In the randomized worst-case setting, the QMC strong tractability conditions of integration for these two spaces are nearly the same. However, in the randomized setting, the sufficient conditions for QMC strong tractability for $\mathcal{H}_{d, \gamma}^{\mathrm{SH}}$ are stronger than those for $\mathcal{H}_{d, \eta, \boldsymbol{\beta}}^{\text {wav }}$ when $\eta=1$. This may arise from the difference between (48) and (52). Note from the proof of Lemma 6 that the upper bound in (52) is not necessarily sharp. If this upper bound can be improved, then the sufficient conditions for strong tractability for $\mathcal{H}_{d, \gamma}^{\mathrm{SH}}$ might be weakened.

\section{ACKNOWLEDGMENTS}

The authors are grateful to Stefan Heinrich, who provided substantial input to the proof of Lemma 6. The authors would also like to thank the referees for their valuable comments and suggestions.

\section{REFERENCES}

[1] Heinrich, S., Hickernell, F. J. and Yue R.-X. (2004). Optimal quadrature for Haar wavelet spaces, Math. Comp. 73, 259-277. MR2034121 (2004k:65037)

[2] Hickernell, F. J. (1996). The mean square discrepancy of randomized nets, ACM Trans. Model. Comput. Simul. 6, 274-296.

[3] Hickernell, F. J. and Hong, H. S. (1999). The asymptotic efficiency of randomized nets for quadrature, Math. Comp., 68, 767-791. MR.1609662 (99i:65021)

[4] Hickernell, F. J. and Wang, X. (2002). The error bounds and tractability of quasi-Monte Carlo algorithms in infinite dimension, Math. Comp., 71, 1641-1661. MR.1933048 (2003i:65009)

[5] Hickernell, F. J. and Woźniakowski, H. (2000). Integration and approximation in arbitrary dimensions, Adv. Comput. Math. 12, 25-58. MR.1758946 (2001d:65017)

[6] Hickernell, F. J. and Woźniakowski, H. (2001a). The price of pessimism for multidimensional quadrature, J. Complexity, 17, 625-659. MR1881662 (2002m:60013)

[7] Hickernell, F. J. and Woźniakowski, H. (2001b). Tractability of multivariate integration for periodic functions, J. Complexity, 17, 660-682. MR1881663(2003g:65028)

[8] Hickernell, F. J. and Yue, R.-X. (2000). The mean square discrepancy of scrambled $(t, s)$ sequences, SIAM J. Numer. Anal. 38, 1089-1112. MR.1786132 (2002c:65009)

[9] Kuo, F. Y. and Sloan, I. H. (2004). Quasi-Monte Carlo methods can be efficient for integration over products of spheres, J. Complexity to appear.

[10] Niederreiter, H. (1988). Low-discrepancy and low-dispersion sequences, J. Number Theory 30, 51-70. MR0960233 (89k:11064)

[11] Niederreiter, H. (1992). Random Number Generation and Quasi-Monte Carlo Methods, SIAM, Philadelphia. MR 1172997 (93h:65008)

[12] Niederreiter, H. and Pirsic, G. (2001). The microstructure of $(t, m, s)$-nets, J. Complexity 17, 683-696. MR 1881664 (2002m:11073)

[13] Owen, A. B. (1995). Randomly permuted $(t, m, s)$-nets and $(t, s)$-sequences, in Monte Carlo and Quasi-Monte Carlo Methods in Scientific Computing (H. Niederreiter and P. J. S. Shiue, eds.), Lecture Notes in Statistics, Vol. 106, Springer-Verlag, 299-317. MR1445791(97k:65013)

[14] Owen, A. B. (1997a). Monte Carlo variance of scrambled equidistribution quadrature, SIAM J. Num. Anal. 34, 1884-1910. MR1472202(98h:65006)

[15] Owen, A. B. (1997b). Scrambled net variance for integrals of smooth functions, Ann. Statist. 25, 1541-1562. MR 1463564 (98j:65004)

[16] Owen, A. B. (1998). Scrambled Sobol and Niederreiter-Xing points, J. Complexity 14, 466489. MR.1659008 (2000c:65005) 
[17] Sloan, I. H. and Woźniakowski, H. (1998). When are quasi-Monte Carlo algorithms efficient for high dimensional integrals, J. Complexity 14, 1-33. MR1617765 (99d:65384)

[18] Sloan, I. H. and Woźniakowski, H. (2001). Tractability of multivariate integration for weighted Korobov classes, J. Complexity 17, 697-721. MR1881665 (2003g:65030)

[19] Sloan, I. H. and Woźniakowski, H. (2004). When does Monte Carlo depend polynomially on the number of variables? in Monte Carlo and Quasi-Monte Carlo Methods 2002 (H. Niederreiter, editor), Springer-Verlag, 407-437. MR2076949

[20] Sobol', I. M. (1967). The distribution of points in a cube and the accurate evaluation of integrals (in Russian), Zh. Vychisl. Mat. i Mat. Phys. 7, 784-802. MR0219238 (36:2321)

[21] Wahba, G. (1990). Spline Models for Observational Data, SIAM, Philadelphia. MR.1045442 (91g:62028)

[22] Wang, X. (2002). A constructive approach to strong tractability using quasi-Monte Carlo algorithms, J. Complexity 18, 683-701. MR1928803(2003m:65008)

[23] Wang, X. (2003). Strong tractability of multivariate integration using quasi-Monte Carlo algorithms, Math. Comp. 72, 823-838. MR1954970(2003m:65009)

[24] Woźniakowski, H. (2000). Efficiency of quasi-Monte Carlo algorithms for high dimensional integrals, In Monte Carlo and Quasi-Monte Carlo Methods 1998 (H. Niederreiter and J. Spanier, eds.), Springer-Verlag, Berlin, pp. 114-136. MR.1849846 (2002e:65015)

[25] Yue, R.-X. and Hickernell, F. J. (2001). Integration and approximation based on scrambled sampling in arbitrary dimensions, J. Complexity 17, 897. MR1881675 (2002m:65005)

[26] Yue, R.-X. (1999). Variance of quadrature over scrambled unions of nets, Statistica Sinica 9, 451-473. MR1707849 (2000h:65022)

[27] Yue, R.-X. and Mao, S.-S. (1999). On the variance of quadrature over scrambled nets and sequences, Stat. and Prob. Letters 44, 267-280. MR1711617 (2000i:65008)

Division of Scientific Computation, E-Institute of Shanghai Universities, 100 Guilin Road, Shanghai 200234, People's Republic of China

E-mail address: yue2@shnu.edu.cn

Department of Applied Mathematics, Shanghai Normal University, Shanghai, PeoPLE's Republic of ChinA

Current address: Department of Applied Mathematics, Illinois Institute of Technology, 10 West 32nd Street, E1 Building, Room 208, Chicago, Illinois 60616-3793

E-mail address: fred@hkbu.edu.hk, hickernell@iit.edu 